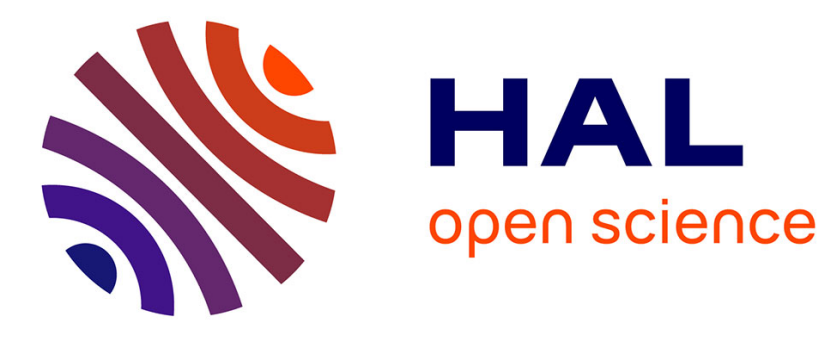

\title{
Field statistics in linear viscoelastic composites and polycrystals.
}

\author{
Claudiu Badulescu, Noel Lahellec, Pierre Suquet
}

\section{To cite this version:}

Claudiu Badulescu, Noel Lahellec, Pierre Suquet. Field statistics in linear viscoelastic composites and polycrystals.. European Journal of Mechanics - A/Solids, 2015, 45, pp.329-344. 10.1016/j.euromechsol.2014.07.012 . hal-01069603

\section{HAL Id: hal-01069603 https://hal.science/hal-01069603}

Submitted on 6 Oct 2014

HAL is a multi-disciplinary open access archive for the deposit and dissemination of scientific research documents, whether they are published or not. The documents may come from teaching and research institutions in France or abroad, or from public or private research centers.
L'archive ouverte pluridisciplinaire HAL, est destinée au dépôt et à la diffusion de documents scientifiques de niveau recherche, publiés ou non, émanant des établissements d'enseignement et de recherche français ou étrangers, des laboratoires publics ou privés. 


\title{
Field statistics in linear viscoelastic composites and polycrystals.
}

\author{
Claudiu Badulescu ${ }^{\mathrm{b}}$, Noel Lahellec ${ }^{\mathrm{a}, *}$, Pierre Suquet $^{\mathrm{a}}$ \\ ${ }^{a}$ Laboratoire de Mécanique et d'Acoustique, \\ CNRS UPR 7051, Aix-Marseille University, Ecole Centrale Marseille \\ 31, Chemin Joseph Aiguier, 13402 Marseille Cedex 20, France \\ ${ }^{b}$ Laboratoire Brestois de Mécanique et des Systèmes, \\ EA 4325, ENSTA Bretagne, Université de Brest, ENIB, UEB \\ 2 rue François Verny, 29806 BREST Cedex 9, France
}

\begin{abstract}
The aim of this study is to estimate the effective response, as well as the statistics of the fields (average and fluctuations per phase) in linear viscoelastic heterogeneous materials. To this effect, a variational method based on a rate variational principle (RVP) has recently been introduced by the authors in which, at each time step, the stress or strain fields are approximated by those of a linear thermoelastic comparison composite. In the present study, a different derivation of this estimate is proposed, based on a simple approximation of the stress field along the time steps. The present study also explores the accuracy of the RVP model by comparing its predictions with reference results, either in closed form (for specific two-phase particulate composites) or obtained by full-field simulations (FFT method) for 2D polycrystals. A differential equation for the second moment of the stress field in the individual phases of two-phase particulate composites is given for the first time. These comparisons show that the RVP model delivers a very accurate estimate of the effective behavior as well as of the statistics of the local fields in two-phase particulate composites. For polycrystalline materials subjected to monotonic loading, the effective behavior and the statistics of the local fields are well predicted. The agreement is less accurate for cycling loadings.
\end{abstract}

Keywords: Homogenization, visco-elasticity, Maxwellian solid, two-phase composite, 2D-polycrystal

\section{Introduction}

This study is devoted to the effective response of composites made from linear viscoelastic phases, more specifically Maxwellian phases, characterized by a relation between the linearized strain $\varepsilon$ and the Cauchy stress $\boldsymbol{\sigma}$ in the form:

$$
\varepsilon=\varepsilon^{\mathrm{e}}+\varepsilon^{\vee}, \quad \varepsilon^{\mathrm{e}}=M^{\mathrm{e}(r)}: \sigma, \quad \dot{\varepsilon}^{\vee}=M^{\vee(r)}: \sigma,
$$

where $\boldsymbol{M}^{\mathrm{e}(r)}$ and $\boldsymbol{M}^{\mathrm{v}(r)}$ denote respectively the elastic and viscous compliance moduli of phase $r$ (with inverse $\boldsymbol{L}^{\mathrm{e}(r)}$ and $\boldsymbol{L}^{\mathrm{v}(r)}$ ) and where an over-dot denotes derivation with respect to time.

The motivation for studying the homogenization of the rather elementary Maxwell model (1) is twofold. First, it is a prerequisite to the study of more physical linear time-dependent models such as the Burger's model or the generalized Maxwell's model which was found by Diani and Gilormini (2014) to capture accurately the behavior of styrene butadiene rubbers. Second, it is

\footnotetext{
*Corresponding author: Tel:+33491164206

Email addresses: claudiu.badulescu@ensta-bretagne.fr (Claudiu Badulescu), lahellec@lma.cnrs-mrs.fr (Noel Lahellec), suquet@lma.cnrs-mrs.fr (Pierre Suquet )
} 
a natural prerequisite to the study of nonlinear viscoelastic composites, where the importance of higher-order statistics of the fields is now well established.

Several Mean Field Homogenization methods (MFH) can be used to predict the effective properties of linear viscoelastic composites. The most classical procedure is based on the correspondence principle. Upon application of the Laplace-Carson transform, the boundary value problem solved by the local stress and strain fields becomes a linear elastic problem in the Laplace domain (Hashin, 1970). Then classical MFH methods can be applied to obtain the effective behavior of the composite in the Laplace domain. The effective relaxation function in the time domain is then obtained by an inverse Laplace-Carson transform. As is well known, even when the individual phases have a "short memory" (such as the Maxwell model (1)), the effective behavior of composites made from different such phases may exhibit a "long memory" effect which manifests itself in the effective constitutive relations through an integral kernel (e.g. Sanchez-Hubert and Sanchez-Palencia, 1978; Laws and Mc Laughlin, 1978; Francfort and Suquet, 1986; Suquet, 1987; Turner and Tomé, 1993; Rougier et al., 1993, among others).

The procedure based on the Laplace transform is convenient when the inverse Laplace transform of the effective complex moduli can be performed in closed form. This is the case for specific forms of these moduli and Laws and Mc Laughlin (1978), Rougier et al. (1993), Masson et al. (2012) derived explicit expressions for the effective creep kernels of several classes of composites or polycrystals with specific microstructures. Unfortunately, and this is a first limitation of the correspondence principle, for other important classes of microstructures (polycrystals with arbitrary microstructure for example), the inversion has to be performed numerically and approximations are required (such as the collocation method Schapery, 1962; Rekik and Brenner, 2011). A second limitation of the correspondence principle is that it does not give access to higher-order statistics of the fields in the different phases, besides the first moment, since the Laplace transforms of the higher-order moments of a field are not the higher-order moments of the Laplace transforms of this field (in contradiction with what is sometimes assumed in the literature). It is actually an open question to find exact relations for the higher-order statistics of fields (stress, strain-rate) in the different constituents of viscoelastic composites, even linear ones. An analytical result is given here for the second moment of the stress field in the specific case of incompressible two-phase composites with a microstructure corresponding to one of the Hashin-Shtrikman bounds. A third limitation of the correspondence principle, actually inherent to all constitutive relations expressed by integral kernels with long memory effects, is that when they are used in a structural computation (by the Finite Element Method for instance), the integration of the constitutive relations at each time step of the global resolution requires the storage of the whole stress (or strain) history along the whole loading path. However, constitutive relations with integral kernels can be equivalently formulated by means of internal variables satisfying first-order differential equations. In specific situations the number of internal variables can be rigorously reduced to a finite number, as shown by Ricaud and Masson (2009) for particulate composites, or can be truncated to a finite number as proposed as an approximation by $\mathrm{Vu}$ et al. (2012).

A different approach to estimate the effective behavior of linear viscoelastic composites, based on the interaction law of Molinari (2002), has been explored by Mercier et al. (2005) who retrieved the exact effective response of two-phase composites with incompressible Maxwellian behavior. Unfortunately, this approach, like the one based on the correspondence principle, cannot estimate the higher-order statistics of the stress (or strain) fields (intraphase fluctuations) which are required for nonlinear constituents by the most recent nonlinear homogenization theories (see Ponte Castañeda, 2002a,b; Idiart et al., 2006; Idiart and Ponte Castañeda, 2007).

Lahellec and Suquet (2007a, 2007b, 2013) have recently proposed two variational approaches to couple elastic and dissipative effects in composite materials. These approaches apply when these two aspects of the behavior of the phases can be described by means of two constitutive potentials 
(within the class of standard generalized materials). The first potential is the free-energy density $w\left(\varepsilon, \varepsilon^{\vee}\right)$. The second potential is the dissipation potential $\varphi\left(\dot{\varepsilon}^{v}\right)$ which describes the evolution of the irreversible mechanisms. Using a discretization in time of the evolution equations, Lahellec and Suquet (2007a); Lahellec and Suquet (2013) have derived an incremental variational principle governing the state of the composite at the end of a time step, assuming that the state at the beginning of the time step is known. A nice feature of these variational principles is that, unlike the correspondence principle, they are not restricted to linear phases. They allowed the authors to extend to phases governed by two potentials some of the approaches initially developed in nonlinear homogenization for phases governed by a single potential (see Lahellec and Suquet, 2007b,c; Lahellec and Suquet, 2013). The two slightly different approches have been applied successfully to elasto-viscoplastic phases (without threshold, sometimes called nonlinear viscoelasticity) subjected to loading-unloading conditions along radial paths. However limitations of the first estimate have appeared for non radial loading conditions, involving rotation of the principal axes of the loading which were not observed (or to a lesser extent) with the second estimate (called RVP since it is based on a rate variational principle). The aim of this paper is to present a simplified version of the RVP estimate and to explore further its capability to predict accurately both the effective response of composites and polycrystals, and the first and second-order statistics of the fields in the individual constituents.

The present paper is organized as follows. In section 2, a simplified and heuristic derivation of the RVP model of Lahellec and Suquet (2013), initially proposed for elasto-(visco)plasticity, is given. This model is applied in section 3 to two-phase particulate composites and in section 4 to $2 \mathrm{D}$ polycrystals. The accuracy of its predictions for the second-order statistics of the fields is shown in section 3 by comparison with the exact expression of the second moment of the stress field (derived in Appendix A) in incompressible two-phase particulate composites.

\section{A simplified version of the variational estimate of Lahellec and Suquet (2013)}

\subsection{Strain-rate formulation}

The composite materials considered in the present study are made from $N$ different homogeneous constituents, or phases, which are assumed to be randomly distributed in a specimen occupying a volume $V$, at a length scale that is much smaller than the size of $V$. Each constituent is linear viscoelastic (and Maxwellian), governed by the constitutive relations (1). Define

$$
\boldsymbol{M}^{\mathrm{e}}(\boldsymbol{x})=\sum_{r=1}^{N} \boldsymbol{M}^{\mathrm{e}(r)} \chi^{(r)}(\boldsymbol{x})
$$

(with a similar definition for $\boldsymbol{M}^{\vee}(\boldsymbol{x})$ ), where $\chi^{(r)}$ is the characteristic function of phase $r$. The volume averages of a function $f$ over the composite $V$ and over phase $r$ are denoted as $\bar{f}=\langle f\rangle=$ $\frac{1}{|V|} \int_{V} f \mathrm{~d} v$ and $\bar{f}^{(r)}=\langle f\rangle_{r}=\frac{1}{\left|V_{r}\right|} \int_{V_{r}} f \mathrm{~d} v$ respectively.

A strain-rate formulation is used and the boundary value problem to be solved to determine the local stress and strain rate fields in the volume element $V$ consists of the equilibrium equations, compatibility conditions, constitutive relations and boundary conditions ${ }^{1}$ :

$$
\dot{\varepsilon}(\boldsymbol{x}, t)=\boldsymbol{M}^{\mathrm{e}}(\boldsymbol{x}): \dot{\boldsymbol{\sigma}}(\boldsymbol{x}, t)+\boldsymbol{M}^{\vee}(\boldsymbol{x}): \boldsymbol{\sigma}(\boldsymbol{x}, t), \quad \operatorname{div} \boldsymbol{\sigma}=0, \quad\langle\dot{\boldsymbol{\varepsilon}}(t)\rangle=\dot{\bar{\varepsilon}}(t) .
$$

\footnotetext{
${ }^{1}$ Boundary conditions are not specified nor discussed here. They are of the classical types, see Suquet (1987) or Ponte Castañeda and Suquet (1998) for more details, ensuring that the validity of Hill's lemma between the stress and strain fields.
} 
The effective behavior of the composite is defined as the relation between the average stress $\overline{\boldsymbol{\sigma}}(t)$ at time $t$ and the history of the average strain before $t, \bar{\varepsilon}(s), 0 \leq s \leq t$.

The time derivative of $\boldsymbol{\sigma}$ in (2) can be approximated by a difference quotient after use of an implicit (backward) Euler-scheme. The time interval of study $[0, T]$ is discretized into time intervals $t_{0}=0, t_{1}, \ldots, t_{n}, t_{n+1}, \ldots, t_{N}=T$. The time step $t_{n+1}-t_{n}$ is denoted by $\Delta t$ (its dependence on $n$ is omitted for simplicity), the value $f\left(t_{n}\right)$ of a function $f$ evaluated at time $t_{n}$ is denoted by $f_{n}$. Assuming that $\boldsymbol{\sigma}_{n}$ is known at time $t_{n}$, the time-discretization procedure applied to (2) leads to the following system in which the strain rate $\dot{\boldsymbol{\varepsilon}}_{n+1}$ is the kinematic unknown :

$$
\left.\begin{array}{c}
\dot{\boldsymbol{\varepsilon}}_{n+1}(\boldsymbol{x})=\frac{1}{\Delta t}\left(\boldsymbol{M}^{\mathrm{e}(r)}+\Delta t \boldsymbol{M}^{\mathrm{v}(r)}\right): \boldsymbol{\sigma}_{n+1}(\boldsymbol{x})-\frac{1}{\Delta t} \boldsymbol{M}^{\mathrm{e}(r)}: \boldsymbol{\sigma}_{n}(\boldsymbol{x}) \text { in phase } r . \\
\operatorname{div} \boldsymbol{\sigma}_{n+1}(\boldsymbol{x})=0, \quad\left\langle\dot{\boldsymbol{\varepsilon}}_{n+1}(\boldsymbol{x})\right\rangle=\dot{\overline{\boldsymbol{\varepsilon}}}_{n+1} .
\end{array}\right\}
$$

This discretized boundary value problem involves a non classical thermoelastic constitutive relation between $\boldsymbol{\sigma}_{n+1}$ and $\dot{\boldsymbol{\varepsilon}}_{n+1}$ with piecewise uniform elastic moduli $\Delta t\left(\boldsymbol{M}^{\mathrm{e}(r)}+\Delta t \boldsymbol{M}^{\mathrm{v}(r)}\right)^{-1}$ but with a non-piecewise uniform eigenstrain $-\boldsymbol{M}^{\mathrm{e}(r)}: \boldsymbol{\sigma}_{n}(\boldsymbol{x})$. Because of the nonuniformity of this eigenstrain, the fields (stress and strain-rate) solutions of (3) cannot be estimated by standard Mean Field Homogenization analytical methods. Indeed, the effective eigenstrain in thermoelastic composites can only be expressed, to the authors' knowledge, conveniently when the eigenstrains are uniform within the different phases. In order to circumvent this difficulty (and to make the eigenstrain piecewise constant), one can replace in (3) $\boldsymbol{\sigma}_{n}(\boldsymbol{x})$ by its average per phase $\left\langle\boldsymbol{\sigma}_{n}(\boldsymbol{x})\right\rangle_{r}$. It will be shown in section 3 that this approximation (denoted RVP1st) is a poor approximation (and an underestimate) to the exact effective response of the composite.

The aim of the present work is precisely to approximate the eigenstrain in (3) by an expression different from the average per phase, still involving a piecewise uniform eigenstrain in order to resort to classical results for thermoelastic composites.

\subsection{Heuristic derivation of the RVP estimate}

It is shown here that the RVP estimate of Lahellec and Suquet (2013) amounts to replacing the actual stress field $\boldsymbol{\sigma}_{n}$ by a stress field $\widehat{\boldsymbol{\sigma}}_{n}$ defined as follows :

$$
\widehat{\boldsymbol{\sigma}}_{n}(\boldsymbol{x})=\boldsymbol{H}^{(r)} \boldsymbol{\sigma}_{n+1}(\boldsymbol{x})+\left(\boldsymbol{I}-\boldsymbol{H}^{(r)}\right): \boldsymbol{\sigma}_{n}^{(r)},
$$

where $\boldsymbol{\sigma}_{n+1}(\boldsymbol{x})$ is unknown, $\boldsymbol{H}^{(r)}$ is an unknown fourth-order tensor (uniform over phase $r$ ) and $\boldsymbol{\sigma}_{n}^{(r)}$ is an unknown second-order tensor (uniform over phase $r$ ). Replacing $\boldsymbol{\sigma}_{n}(\boldsymbol{x})$ by $\widehat{\boldsymbol{\sigma}}_{n}(\boldsymbol{x})$ in (3) leads to the following boundary value problem:

$$
\boldsymbol{\sigma}=\mathcal{L}_{0}^{(r)}: \dot{\boldsymbol{\varepsilon}}+\boldsymbol{\tau}_{0}^{(r)} \quad \text { in phase } \quad r, \quad \operatorname{div} \boldsymbol{\sigma}(\boldsymbol{x})=0, \quad\langle\dot{\boldsymbol{\varepsilon}}\rangle=\dot{\bar{\varepsilon}},
$$

where $\dot{\boldsymbol{\varepsilon}}$ and $\boldsymbol{\sigma}$ stand for $\dot{\boldsymbol{\varepsilon}}_{n+1}$ and $\boldsymbol{\sigma}_{n+1}$ in (3) and

$$
\left.\begin{array}{l}
\boldsymbol{M}_{0}^{\mathrm{e}(r)}=\boldsymbol{M}^{\mathrm{e}(r)}:\left(\boldsymbol{I}-\boldsymbol{H}^{(r)}\right), \quad \mathcal{L}_{0}^{(r)}=\Delta t\left(\boldsymbol{M}_{0}^{\mathrm{e}(r)}+\Delta t \boldsymbol{M}^{\mathrm{v}(r)}\right)^{-1}, \\
\boldsymbol{\tau}_{0}^{(r)}=\left(\boldsymbol{M}_{0}^{\mathrm{e}(r)}+\Delta t \boldsymbol{M}^{\mathrm{v}(r)}\right)^{-1}: \boldsymbol{M}_{0}^{\mathrm{e}(r)}: \boldsymbol{\sigma}_{n}^{(r)} .
\end{array}\right\}
$$

The problem (5) is now a boundary value problem for a linear thermoelastic comparison composite with uniform elastic moduli $\mathcal{L}_{0}^{(r)}$ and uniform eigenstrain $-1 / \Delta t \boldsymbol{M}_{0}^{\mathrm{e}(r)}: \boldsymbol{\sigma}_{n}^{(r)}$ in phase $r$. Standard homogenization results for $N$-phase thermoelastic composites can therefore be used to estimate the effective behavior and the statistics of the fields $\dot{\boldsymbol{\varepsilon}}(\boldsymbol{x}), \boldsymbol{\sigma}(\boldsymbol{x})$ solution of (5). 
It remains to specify how $\boldsymbol{H}^{(r)}$ and $\boldsymbol{\sigma}_{n}^{(r)}$ are chosen. If they could be chosen in such a way that the fields $\boldsymbol{\sigma}_{n}(\boldsymbol{x})$ and $\widehat{\boldsymbol{\sigma}}_{n}(\boldsymbol{x})$ would coincide at every material point $\boldsymbol{x}$, then the problems (3) and (5) would be equivalent. Unfortunately this equality cannot be achieved pointwisely in general. It is proposed to relax the equality between the fields $\widehat{\boldsymbol{\sigma}}_{n}$ and $\boldsymbol{\sigma}_{n}$, in the sense that it is no more required that they coincide at every material point, but only that they share the same first and second-order statistics per phase :

$$
\left\langle\boldsymbol{\sigma}_{n}\right\rangle_{r}=\left\langle\widehat{\boldsymbol{\sigma}}_{n}\right\rangle_{r}
$$

and

$$
\left\langle\boldsymbol{\sigma}_{n} \otimes \boldsymbol{\sigma}_{n}\right\rangle_{r}=\left\langle\widehat{\boldsymbol{\sigma}}_{n} \otimes \widehat{\boldsymbol{\sigma}}_{n}\right\rangle_{r} .
$$

The equality between the first and second moments of $\boldsymbol{\sigma}_{n}$ and $\hat{\boldsymbol{\sigma}}_{n}$ in phase $r$ can be re-written as the equality between the first moment and the fluctuations of these fields. Following Idiart and Ponte Castañeda (2007) the (quadratic) fluctuations of a field $\boldsymbol{\sigma}$ in phase $r$ is defined as

$$
\boldsymbol{C}^{(r)}(\boldsymbol{\sigma})=\left\langle\left(\boldsymbol{\sigma}-\overline{\boldsymbol{\sigma}}^{(r)}\right) \otimes\left(\boldsymbol{\sigma}-\overline{\boldsymbol{\sigma}}^{(r)}\right)\right\rangle_{r}, \quad \overline{\boldsymbol{\sigma}}^{(r)}=\langle\boldsymbol{\sigma}\rangle_{r} .
$$

According to (4), $\boldsymbol{\sigma}$ is the only field contributing to the fluctuations of $\hat{\boldsymbol{\sigma}}_{n}$ and therefore (8) can be replaced by a relation between the fluctuations of $\boldsymbol{\sigma}_{n}$ and the fluctuations of $\boldsymbol{\sigma}$. Then, $\boldsymbol{\sigma}_{n}^{(r)}$ and $\boldsymbol{H}^{(r)}$ solve the two following equations:

$$
\begin{array}{r}
\left\langle\boldsymbol{\sigma}_{n}\right\rangle_{r}=\boldsymbol{H}^{(r)}\langle\boldsymbol{\sigma}\rangle_{r}+\left(\boldsymbol{I}-\boldsymbol{H}^{(r)}\right): \boldsymbol{\sigma}_{n}^{(r)}, \\
\boldsymbol{C}^{(r)}\left(\boldsymbol{\sigma}_{n}\right)=\boldsymbol{C}^{(r)}\left(\boldsymbol{H}^{(r)}: \boldsymbol{\sigma}\right),
\end{array}
$$

where $\boldsymbol{\sigma}$ is the solution of the boundary value problem (5). It enters (10) and (11) through its first and second-order statistics which depend in a nonlinear (and implicit) way on $\boldsymbol{\sigma}_{n}^{(r)}$ and $\boldsymbol{H}^{(r)}$. In summary, assuming that the first moment and the fluctuations of the field $\boldsymbol{\sigma}_{n}$ at time $t_{n}$ are known in each individual phase, $r$, the resolution of the system of equations (10) and (11) allows for the determination of $\boldsymbol{\sigma}_{n}^{(r)}$ and $\boldsymbol{H}^{(r)}$. Then the first moment and the fluctuations of the new field $\sigma$ at time $t_{n+1}$, solution of the linear thermoelastic problem (5), can be determined by standard relations in thermoelastic composites. Note however, that the system of equations (10) and (11) which is to be solved for $\boldsymbol{\sigma}_{n}^{(r)}$ and $\boldsymbol{H}^{(r)}$ is nonlinear, and can have several roots. It is solved numerically and details on the numerical procedure are given in Appendix B, under the additional simplification that $\boldsymbol{H}^{(r)}$ can be defined using a single scalar $h^{(r)}$.

\subsection{Variational justification}

Lahellec and Suquet (2013) originally derived the RVP estimate of section 2.2 through a variational principle. The connection with this original derivation can be made as follows. The equations defining the problem (3) are the Euler-Lagrange equations of the variational problem :

$$
\tilde{d}(\dot{\bar{\varepsilon}})=\operatorname{Inf}_{\langle\dot{\varepsilon}\rangle=\dot{\bar{\varepsilon}}}\left\langle\operatorname{Inf}_{\dot{\varepsilon}^{\vee}} \mathcal{D}\left(\dot{\varepsilon}, \dot{\varepsilon}^{\vee}\right)\right\rangle
$$

with $\varepsilon^{\vee}$ the viscous strain and $\mathcal{D}$ is the rate-potential defined for a Maxwell solid by :

$$
\mathcal{D}\left(\dot{\varepsilon}, \dot{\varepsilon}^{\vee}\right)=\frac{\Delta t}{2}\left(\dot{\varepsilon}-\dot{\varepsilon}^{v}\right): L^{\mathrm{e}}:\left(\dot{\varepsilon}-\dot{\varepsilon}^{v}\right)+\sigma_{n}:\left(\dot{\varepsilon}-\dot{\varepsilon}^{v}\right)+\frac{1}{2} \dot{\varepsilon}^{\vee}: L^{\vee}: \dot{\varepsilon}^{v} .
$$

Then, using the variational procedure of Ponte Castañeda (1992) the following estimate for the effective potential $\tilde{d}$ is obtained :

$$
\tilde{d}(\dot{\bar{\varepsilon}}) \approx \operatorname{Inf}_{\langle\dot{\varepsilon}\rangle=\dot{\bar{\varepsilon}}}\left[\left\langle\operatorname{Inf}_{\dot{\varepsilon}^{\vee}} \mathcal{D}_{0}\left(\dot{\varepsilon}, \dot{\varepsilon}^{\vee}\right)\right\rangle+\left\langle\operatorname{Stat}_{\dot{\varepsilon}^{\vee}} \Delta \mathcal{D}\left(\dot{\varepsilon}, \dot{\varepsilon}^{\vee}\right)\right\rangle\right],
$$


where $\mathcal{D}_{0}$ is the rate-potential for a linear comparison composite defined by :

$$
\mathcal{D}_{0}^{(r)}\left(\dot{\varepsilon}, \dot{\varepsilon}^{v}\right)=\frac{\Delta t}{2}\left(\dot{\varepsilon}-\dot{\varepsilon}^{v}\right): \boldsymbol{L}_{0}^{\mathrm{e}(r)}:\left(\dot{\varepsilon}-\dot{\varepsilon}^{v}\right)+\boldsymbol{\sigma}_{n}^{(r)}:\left(\dot{\varepsilon}-\dot{\varepsilon}^{\vee}\right)+\frac{1}{2} \dot{\varepsilon}^{\vee}: \boldsymbol{L}^{\mathrm{v}(r)}: \dot{\varepsilon}^{\vee},
$$

and $\Delta \mathcal{D}=\mathcal{D}-\mathcal{D}_{0}$. The rate-potential $\mathcal{D}_{0}^{(r)}$ involves the reference stress tensor $\boldsymbol{\sigma}_{n}^{(r)}$, already introduced in (4), and the reference elastic stiffness tensor $\boldsymbol{L}_{0}^{\mathrm{e}(r)}$ which is the inverse of $\boldsymbol{M}_{0}^{\mathrm{e}(r)}$ introduced in (6) and which can be related to $\boldsymbol{H}^{(r)}$ by :

$$
\boldsymbol{L}_{0}^{\mathrm{e}(r)}=\left(\boldsymbol{I}-\boldsymbol{H}^{(r)}\right)^{-1}: \boldsymbol{L}^{\mathrm{e}(r)}
$$

The infimum problem in (14) is equivalent to (5). Finally, the reference tensors $\boldsymbol{\sigma}_{n}^{(r)}$ and $\boldsymbol{L}_{0}^{\mathrm{e}(r)}$ are chosen so that the homogenized potential $\tilde{d}$ is stationary :

$$
\tilde{d}(\dot{\bar{\varepsilon}}) \approx \operatorname{Stat}_{\boldsymbol{\sigma}_{n}^{(r)}, \boldsymbol{L}_{0}^{\mathrm{e}(r)}}\left\{\operatorname{Inf}_{\langle\dot{\boldsymbol{\varepsilon}}\rangle=\dot{\bar{\varepsilon}}}\left[\left\langle\operatorname{Inf}_{\dot{\boldsymbol{\varepsilon}}^{\vee}} \mathcal{D}_{0}\left(\dot{\boldsymbol{\varepsilon}}, \dot{\boldsymbol{\varepsilon}}^{\vee}\right)\right\rangle+\left\langle\operatorname{Stat}_{\dot{\boldsymbol{\varepsilon}}^{\vee}} \Delta \mathcal{D}\left(\dot{\varepsilon}, \dot{\varepsilon}^{\vee}\right)\right\rangle\right]\right\} .
$$

These two stationarity conditions are equivalent to (7) and (8) which shows that the two approaches (heuristic and variational) are equivalent.

Lahellec and Suquet (2013) also showed that if $\boldsymbol{L}_{0}^{\mathrm{e}(r)}$ is chosen proportional to $\boldsymbol{L}^{\mathrm{e}(r)}\left(\right.$ ie $\boldsymbol{L}_{0}^{\mathrm{e}(r)}=$ $\theta^{(r)} \boldsymbol{L}^{\mathrm{e}(r)}$ with $\theta^{(r)}$ a scalar variable) or equivalently $\boldsymbol{H}^{(r)}$ is chosen proportional the identity fourthorder tensor (ie $\boldsymbol{H}^{(r)}=h^{(r)} \boldsymbol{I}$ with $h^{(r)}=1-\theta^{(r)^{-1}}$ ) the equation (11) reduces to :

$$
\boldsymbol{C}^{(r)}\left(\boldsymbol{\sigma}_{n}\right):: \boldsymbol{L}^{\mathrm{e}(r)^{-1}}=\boldsymbol{C}^{(r)}\left(\boldsymbol{H}^{(r)}: \boldsymbol{\sigma}\right):: \boldsymbol{L}^{\mathrm{e}(r)^{-1}}
$$

\section{Two-phase particulate composites}

The accuracy of the RVP is assessed in this section for two-phase materials composed of a matrix reinforced by isotropically distributed spherical inclusions. In addition, their microstructure is such that their linear (either purely elastic or purely viscous) properties are accurately described by the Hashin-Shtrikman estimate (with the reference medium being the actual matrix).

\subsection{The RVP method for composites with isotropic phases} by :

Consider a composite with isotropic phases with elastic and viscous properties characterized

$$
\boldsymbol{L}^{\mathrm{e}(r)}=2 \mu^{(r)} \boldsymbol{K}+3 k^{(r)} \boldsymbol{J} \quad \text { and } \quad \boldsymbol{L}^{\mathrm{v}(r)}=2 \eta^{(r)} \boldsymbol{K}+\infty \boldsymbol{J}
$$

where $\boldsymbol{J}$ and $\boldsymbol{K}$ are the usual fourth-order projectors on hydrostatic and deviatoric symmetric second-order tensors, $k^{(r)}$ and $\mu^{(r)}$ are the bulk and the shear elastic moduli of phase $(r)$ and $\eta^{(r)}$ its viscosity (note that this particular form of $\boldsymbol{L}^{\mathrm{v}(r)}$ enforces incompressibility of the viscous strain). For simplicity, the tensor $\boldsymbol{H}^{(r)}$ is chosen proportional to the identity tensor :

$$
\boldsymbol{H}^{(r)}=h^{(r)} \boldsymbol{I}
$$

The different tensors entering (6) take the form :

$$
\left.\begin{array}{rl}
\mathcal{L}_{0}^{(r)} & =2 \mu_{\Delta, 0}^{(r)} \boldsymbol{K}+3 k_{\Delta, 0}^{(r)} \boldsymbol{J}, \\
\mu_{\Delta, 0}^{(r)} & =\frac{\mu^{(r)} \eta^{(r)}}{\mu^{(r)}+\eta^{(r)}\left(1-h^{(r)}\right) / \Delta t}, \quad k_{\Delta, 0}^{(r)}=\frac{k^{(r)}}{1-h^{(r)}} \\
\boldsymbol{\tau}_{0}^{(r)} & =\left(\frac{\eta^{(r)}\left(1-h^{(r)}\right) / \Delta t}{\mu^{(r)}+\eta^{(r)}\left(1-h^{(r)}\right) / \Delta t} \boldsymbol{K}+\boldsymbol{J}\right): \boldsymbol{\sigma}_{n}^{(r)} \cdot
\end{array}\right\}
$$


$\boldsymbol{\sigma}_{n}^{(r)}$ and $h^{(r)}$ are determined by the closure relations (10) and (17). Given the choice (19), these relations become respectively

$$
\left.\begin{array}{c}
\left\langle\boldsymbol{\sigma}_{n}\right\rangle_{r}=h^{(r)}\langle\boldsymbol{\sigma}\rangle_{r}+\left(1-h^{(r)}\right) \boldsymbol{\sigma}_{n}^{(r)}, \\
\boldsymbol{C}^{(r)}\left(\boldsymbol{\sigma}_{n}\right)::\left(\frac{\boldsymbol{K}}{2 \mu^{(r)}}+\frac{\boldsymbol{J}}{3 k^{(r)}}\right)=\left(h^{(r)}\right)^{2} \boldsymbol{C}^{(r)}(\boldsymbol{\sigma})::\left(\frac{\boldsymbol{K}}{2 \mu^{(r)}}+\frac{\boldsymbol{J}}{3 k^{(r)}}\right) \cdot
\end{array}\right\}
$$

Keeping in mind the comments made at the end of section 2.2 , it is reminded that the system (21) is nonlinear in $h^{(r)}$ since $\langle\boldsymbol{\sigma}\rangle_{r}$ and $\boldsymbol{C}^{(r)}(\boldsymbol{\sigma})$ depend on $h^{(r)}$. The resolution of this nonlinear system is discussed in Appendix B. It is shown in figure B.10 that, in certain circumstances, the second equation in (21) may have several roots. A heuristic procedure for determining the most physical root is then proposed for two-phase composites.

Remark : The simplicity of the choice (19) with only one scalar unknown could be found too restrictive. Other forms for the tensor $\boldsymbol{H}^{(r)}$ have been explored and in particular the isotropic decomposition $\boldsymbol{H}^{(r)}=h_{J}^{(r)} \boldsymbol{J}+h_{K}^{(r)} \boldsymbol{K}$ with 2 scalar unknowns $h_{J}^{(r)}$ and $h_{K}^{(r)}$. The resulting nonlinear problem was more complex but no significant improvement was observed (and the results are consequently not reported here).

\subsection{Incompressible phases}

We address first the case where both phases are viscoelastic and incompressible for which an explicit solution for the effective response as well as for the first and second moments of the fields in the individual phases is available in closed form (see Appendix A). Two sets of data have been considered. In the first set of data, the inclusions (phase 1) are stiffer than the matrix, both in the elastic and in the viscous regime.

$$
c^{(1)}=0.4, \mu^{(1)}=30 \mathrm{Gpa}, \quad \mu^{(2)}=1 \mathrm{Gpa}, \quad \eta^{(1)}=6 \text { Gpa.s, } \quad \eta^{(2)}=2 \text { Gpa.s. }
$$

In the second set of data, the contrast between the phases is inverted between the elastic regime and the viscous regime (the inclusions are elastically stiffer than the matrix but their viscosity is lower):

$$
c^{(1)}=0.4, \mu^{(1)}=30 \mathrm{Gpa}, \quad \mu^{(2)}=1 \mathrm{Gpa}, \quad \eta^{(1)}=2 \text { Gpa.s, } \quad \eta^{(2)}=6 \text { Gpa.s. }
$$

The problem is tridimensional. The composite is sheared alternatively at macroscopic strain-rate with constant absolute value but with a change in sign between two subsequent time periods :

$$
\dot{\bar{\varepsilon}}= \pm \dot{\bar{\varepsilon}}_{0}\left(e_{1} \otimes e_{2}+e_{2} \otimes e_{1}\right)
$$

with $\dot{\bar{\varepsilon}}_{0}=210^{-3} \mathrm{~s}^{-1}$. The average stress in the matrix and in the inclusions is also a pure shear parallel to the applied macroscopic shear. The predictions of the RVP model are compared in figure 1 with the exact solution derived in Appendix A. The estimate RVP1st, obtained by replacing directly in (3) $\boldsymbol{\sigma}_{n}(\boldsymbol{x})$ by its average per phase $\left\langle\boldsymbol{\sigma}_{n}(\boldsymbol{x})\right\rangle_{r}$, is also shown. The agreement of the RVP prediction with the exact result is excellent, not only for the effective response but also for the first and second moments (and fluctuations) of the stress field in the matrix (there is no stress fluctuations in the inclusions according to the Hashin-Shtrikman estimate). By contrast the predictions of the RVP1st estimate are not accurate.

\section{Influence of the time step}

The influence of the time-step $\Delta t$ on the accuracy of the predictions is discussed in figure 2 . As can be seen, both the effective response and the fluctuations converge quite rapidly when the time step is reduced. It is recalled that the backward Euler scheme chosen to discretize in time the constitutive equations is implicit and inconditionally stable. Typically 200 time steps are sufficient to capture accurately the loading branch of the effective response. 


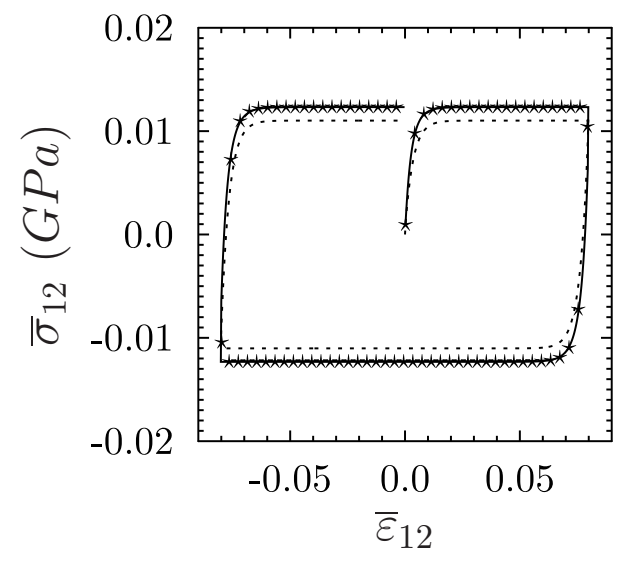

(a)

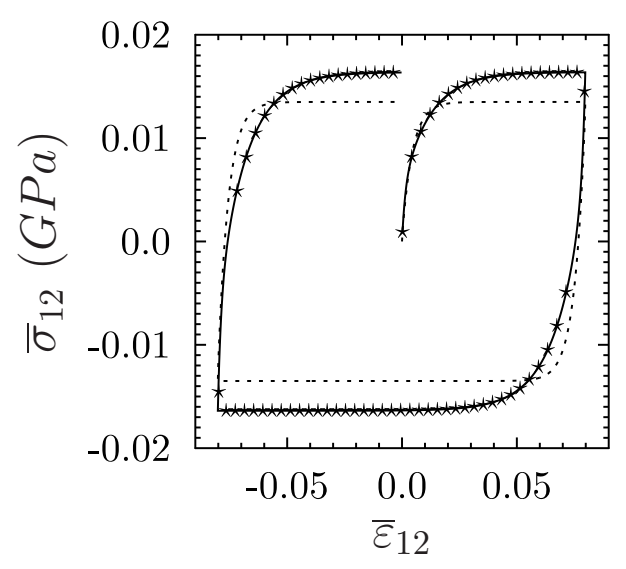

(c)

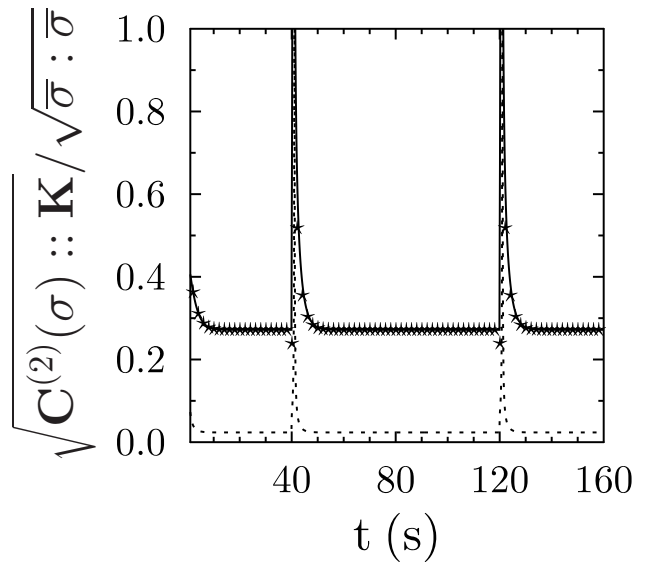

(b)

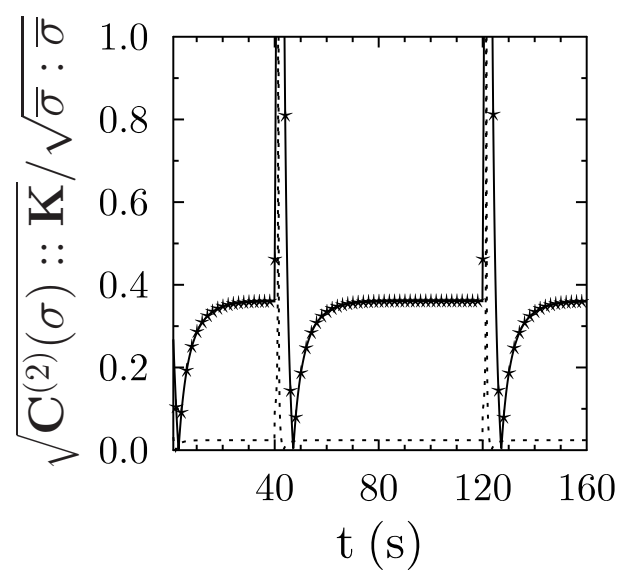

(d)

Figure 1: Incompressible particulate composite subject to cyclic shearing. (a) and (b): material data (22). (c) and (d): material data (23). Comparison between the RVP estimate (solid line), RVP1st estimate (dashed line) and the exact solution of Appendix A (symbol). Left: effective response (macroscopic stress as a function of the macroscopic strain). Right: fluctuations of the stress field in the matrix as a function of time $t$. 


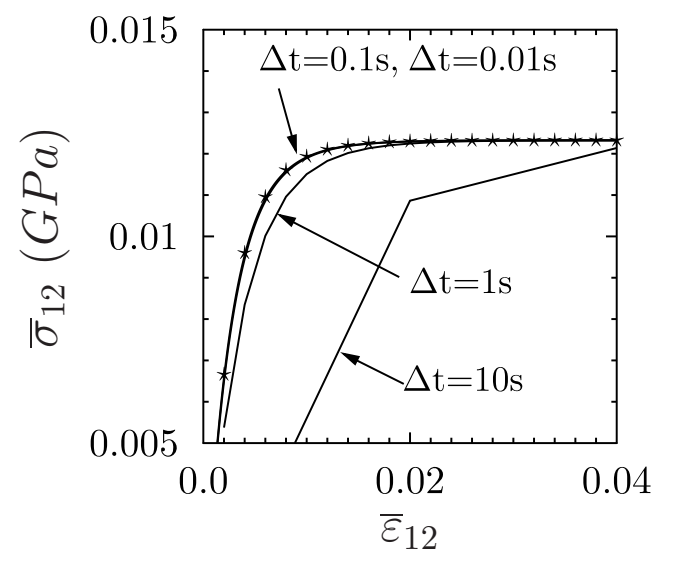

(a)

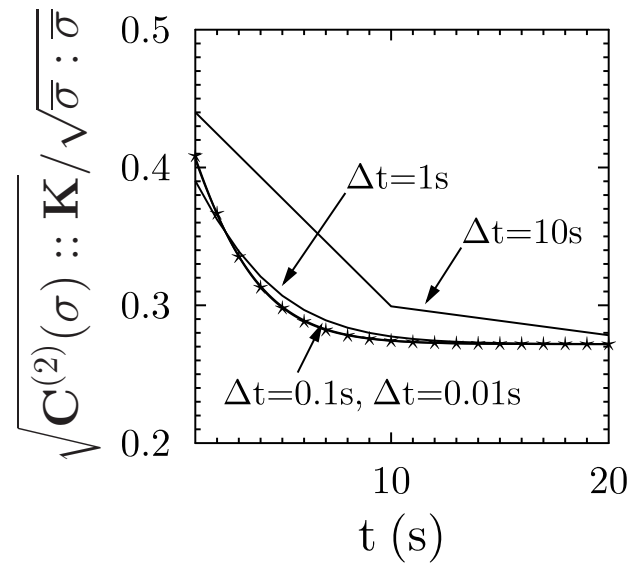

(b)

Figure 2: Incompressible particulate composite under shear. Influence of the time increment $\Delta t$. Material data (22). Comparison between the RVP model (solid line) for different time increments and the exact solution of Appendix A (symbol). Left: effective response (macroscopic stress as a function of the macroscopic strain). Right: fluctuations of the stress field in the matrix as a function of time $t$.

\subsection{Compressible phases}

We consider now a composite made of a compressible viscoelastic matrix reinforced by compressible elastic particles. Again the microstructure of the composite is such that the linear (elastic or viscous) properties of the composite are accurately described by the Hashin-Shtrikman estimate. The effective response of such composites can be obtained by a slight modification of the work of Ricaud and Masson (2009) (these authors assume that both phases have identical elastic properties, but their result can be easily extended to phases with different elastic properties).

Two volume fractions of inclusions and two relaxation times for the matrix are considered. The material data are as follows:

- Inclusion : $c^{(1)}=0.17$ or $0.4, \mu^{(1)}=6 G P a$ and $k^{(1)}=20 G P a$,

- Matrix: $\mu^{(2)}=3 G P a, k^{(2)}=10 G P a$ and $\tau^{(2)}=\frac{\eta^{(2)}}{\mu^{(2)}}=1 s$ or $10 s$.

The composite is subjected to two different loading conditions. First, it is subjected to an isochoric deformation, alternatively in tension and compression, at a macroscopic strain-rate with constant absolute value but with a change in sign between two subsequent time periods:

$$
\dot{\bar{\varepsilon}}(t)= \pm \dot{\bar{\varepsilon}}_{0} \boldsymbol{E}_{0}, \boldsymbol{E}_{0}=\boldsymbol{e}_{1} \otimes \boldsymbol{e}_{1}-\frac{1}{2} \boldsymbol{e}_{2} \otimes \boldsymbol{e}_{2}-\frac{1}{2} \boldsymbol{e}_{3} \otimes \boldsymbol{e}_{3}, \dot{\bar{\varepsilon}}_{0}=5.10^{-2} \mathrm{~s}^{-1} .
$$

The corresponding predictions of the RVP model are compared in Figure 3 with the exact response of the composite (obtained by extending the results of Ricaud and Masson) for two different volume fractions of inclusions and two different relaxation times for the matrix. The agreement is again seen to be very satisfactory.

The second loading involves a rotation of the principal axes of the overall deformation:

$$
\bar{\varepsilon}(t)=\bar{\varepsilon}_{1}\left(\sin \omega t \boldsymbol{E}_{0}+(1-\cos \omega t) \boldsymbol{E}_{1}\right),
$$




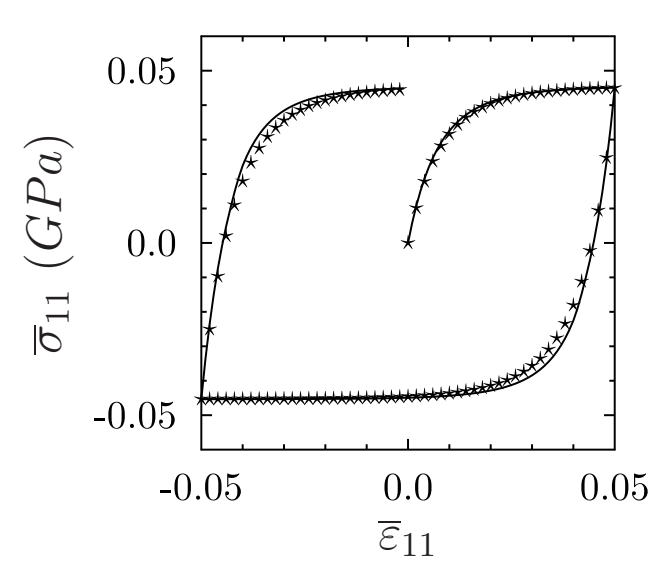

(a)

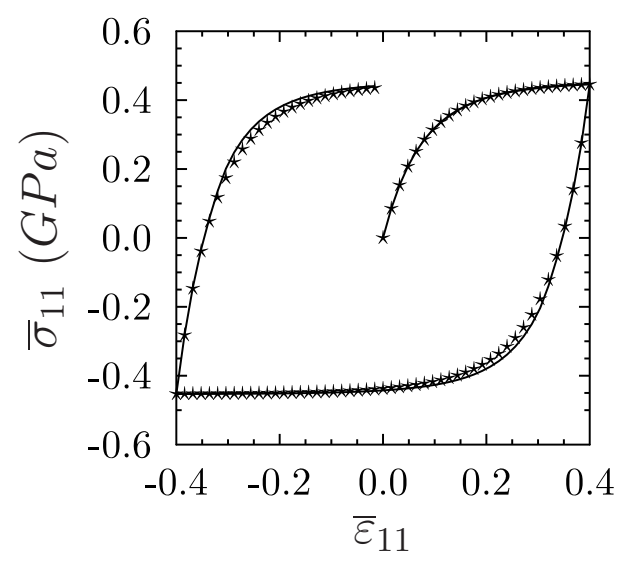

(c)

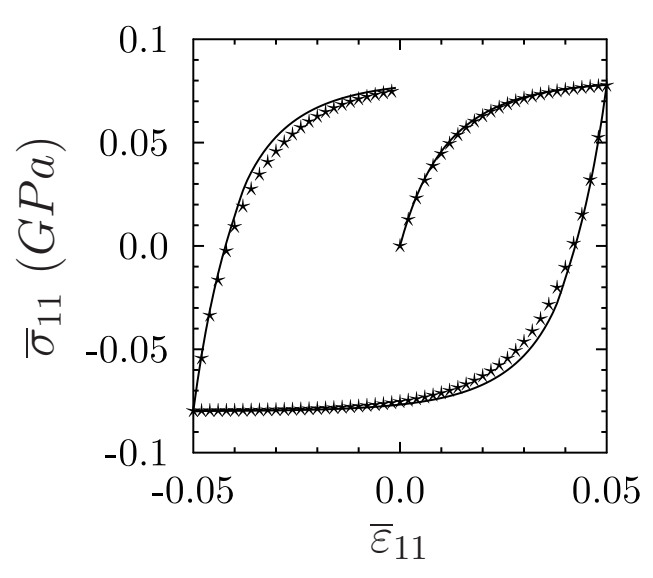

(b)

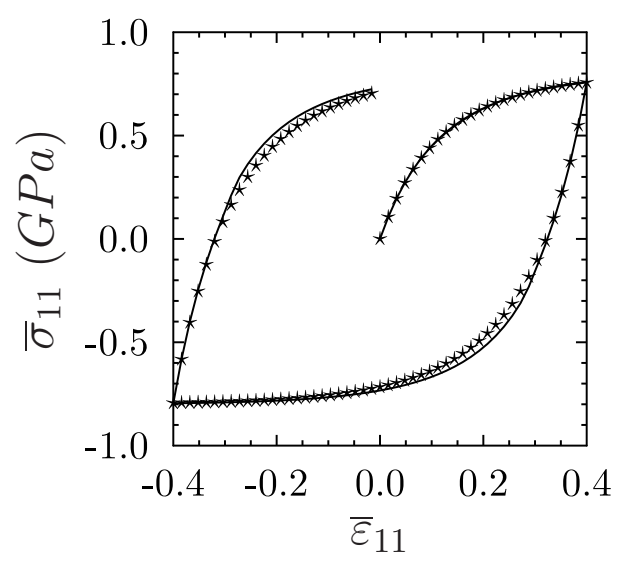

(d)

Figure 3: Compressible particulate composite. Cyclic loading condition (24). Effective response of the composite: exact response (symbols) and predictions of the RVP model (solid line). (a): $c^{(1)}=0.17$ and $\tau^{(2)}=1 s,(\mathrm{~b})$ : $c^{(1)}=0.4$ and $\tau^{(2)}=1 s,(\mathrm{c}): c^{(1)}=0.17$ and $\tau^{(2)}=10 \mathrm{~s}$ and $(\mathrm{d}): c^{(1)}=0.4$ and $\tau^{(2)}=10 \mathrm{~s}$. 


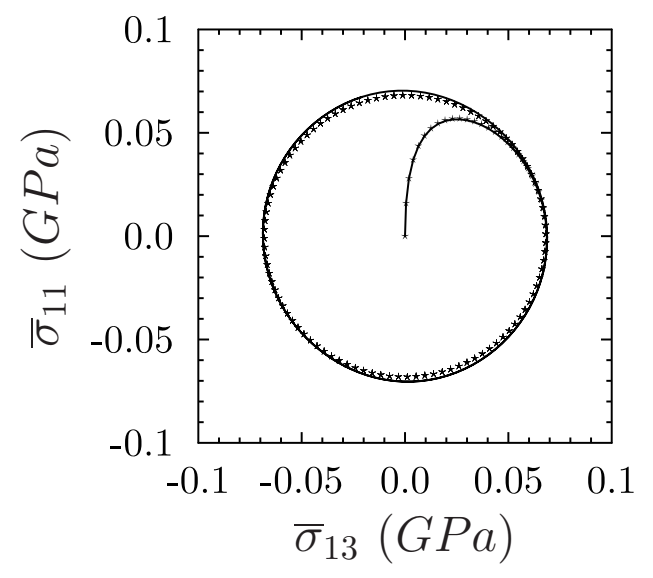

(a)

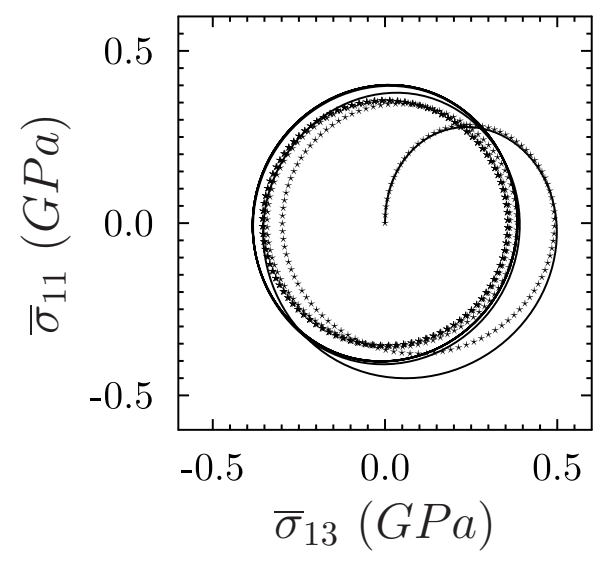

(c)

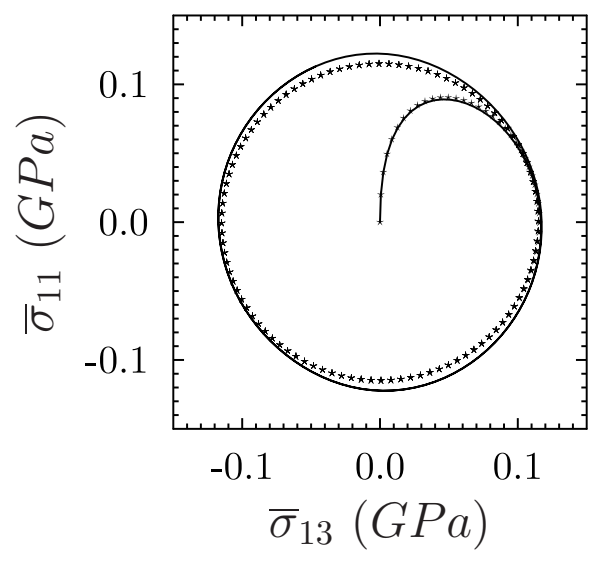

(b)

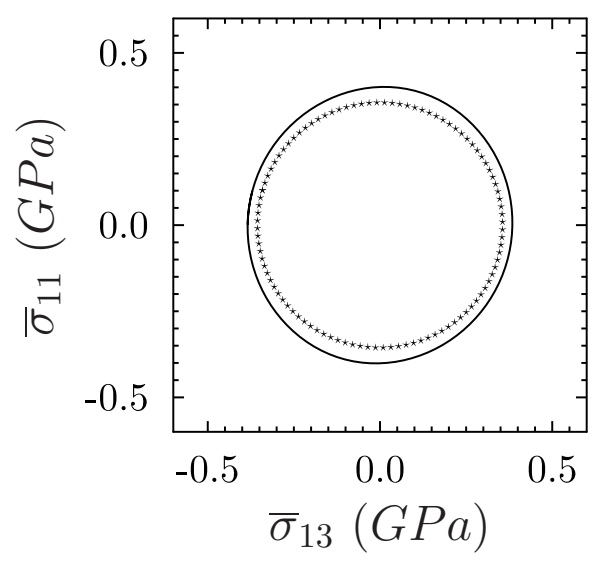

(d)

Figure 4: Rotating loading (25). Effective response of the composite: exact response (symbols) and predictions of the RVP model (solid line). (a): $c^{(1)}=0.17, \tau^{(2)}=1 s$ and $\omega=\frac{\pi}{20}$, (a) (b): $c^{(1)}=0.4, \tau^{(2)}=1 s$ and $\omega=\frac{\pi}{20},(\mathrm{c}): c^{(1)}=0.4, \tau^{(2)}=10 s$ and $\omega=\frac{\pi}{20}$ and $(\mathrm{d}): c_{1}=0.4, \tau^{(2)}=10 \mathrm{~s}$ and $\omega=\frac{\pi}{20}$ for the last cycle. 
where $\boldsymbol{E}_{0}$ is defined in (24),

$$
\boldsymbol{E}_{1}=\frac{1}{2}\left(\boldsymbol{e}_{1} \otimes \boldsymbol{e}_{2}+\boldsymbol{e}_{2} \otimes \boldsymbol{e}_{1}+\boldsymbol{e}_{1} \otimes \boldsymbol{e}_{3}+\boldsymbol{e}_{3} \otimes \boldsymbol{e}_{1}\right),
$$

and

$$
\bar{\varepsilon}_{1}=510^{-2} \text { and } \omega=\frac{\pi}{20}{\mathrm{rad} . \mathrm{s}^{-1} .}^{-}
$$

The predictions of the RVP model are compared with the exact reference results in figure 34 . Again the agreement is seen to be good. A more detailed examination of these two loading cases leads to the following observations :

1. In all cases, the RVP model tends to overestimate the exact response of the composite (the model is a bit too stiff).

2. In the first loading the RVP model coincides with the exact result in the purely elastic and purely viscous limits. The error is maximum in the transient regime with a maximum of $11 \%$ when the volume fraction of inclusions is large and when the relaxation time of the matrix is large $\left(c^{(1)}=0.4\right.$ and $\tau^{(2)}=10 s$ see Figure $\left.3(\mathrm{~d})\right)$.

3. The RVP model is less accurate for the second loading conditions but still captures correctly the rotation of the loading axes. The error increases with the inclusion volume fraction $c^{(1)}$ and with the matrix relaxation time $\tau^{(2)}$ with a maximum of $10 \%$ for the stabilized cycle when $c^{(1)}=0.4, \tau^{(2)}=10 s$ see Figure $4(\mathrm{~d})$.

\section{2D polycrystals}

In this section the RVP estimate derived in section 2.2 is applied to $2 \mathrm{D}$ polycrystals. In a crystal with $K$ slip systems, the viscoelastic (Maxwell type) constitutive relation reads (in the crystal coordinates) :

$$
\boldsymbol{\varepsilon}=\varepsilon^{\mathrm{e}}+\boldsymbol{\varepsilon}^{\mathrm{v}}, \quad \boldsymbol{\varepsilon}^{\mathrm{e}}=\boldsymbol{M}^{\mathrm{e}}: \boldsymbol{\sigma}, \quad \dot{\boldsymbol{\varepsilon}}^{\mathrm{v}}=\sum_{k=1}^{K} \dot{\gamma}_{(k)} \boldsymbol{\mu}_{(k)}, \quad \dot{\gamma}_{(k)}=\dot{\gamma}_{0,(k)} \frac{\boldsymbol{\sigma}: \boldsymbol{\mu}_{(k)}}{\sigma_{0,(k)}} .
$$

$\boldsymbol{\mu}_{(k)}$ and $\gamma_{(k)}$ denote respectively the Schmid tensor of the $k$-th system and the slip along this system:

$$
\boldsymbol{\mu}_{(k)}=\frac{1}{2}\left(\boldsymbol{m}_{(k)} \otimes \boldsymbol{n}_{(k)}+\boldsymbol{n}_{(k)} \otimes \boldsymbol{m}_{(k)}\right),
$$

where $\boldsymbol{n}_{(k)}$ and $\boldsymbol{m}_{(k)}$ denote respectively the unit vectors orthogonal to the slip plane and parallel to the slip direction of the $k$-th system. $\sigma_{0,(k)}$ et and $\dot{\gamma}_{0,(k)}$ denoted respectively the threshold stress and a reference slip rate which define the viscosity of the kth slip system. In the laboratory frame, these relations read in grain $r$ :

$$
\boldsymbol{\varepsilon}=\varepsilon^{\mathrm{e}}+\boldsymbol{\varepsilon}^{\vee}, \quad \boldsymbol{\varepsilon}^{\mathrm{e}}=\boldsymbol{M}^{\mathrm{e}(r)}: \boldsymbol{\sigma}, \quad \dot{\boldsymbol{\varepsilon}}^{\vee}=\sum_{k=1}^{K} \dot{\gamma}_{(k)} \boldsymbol{\mu}_{(k)}^{(r)}, \quad \dot{\gamma}_{(k)}=\dot{\gamma}_{0,(k)} \frac{\boldsymbol{\sigma}: \boldsymbol{\mu}_{(k)}^{(r)}}{\sigma_{0,(k)}} .
$$

$\boldsymbol{M}^{\mathrm{e}(r)}$ and $\boldsymbol{\mu}_{(k)}^{(r)}$ are obtained from the tensors $\boldsymbol{M}^{\mathrm{e}}$ and $\boldsymbol{\mu}$ by rotation :

$$
\boldsymbol{M}^{\mathrm{e}(r)}=\boldsymbol{Q}^{T(r)} \boldsymbol{Q}^{T(r)} \boldsymbol{M}^{\mathrm{e}} \boldsymbol{Q}^{(r)} \boldsymbol{Q}^{(r)} \quad \text { and } \quad \boldsymbol{\mu}_{(k)}^{(r)}=\boldsymbol{Q}^{T(r)} \boldsymbol{\mu}_{(k)} \boldsymbol{Q}^{(r)}
$$

where $\boldsymbol{Q}^{(r)}$ defines the orientation of grain $(r)$. For the special class of polycrystals consisting of columnar orthorhombic grains (see Liu and Ponte Castañeda, 2004) all the grains are cylindrical with parallel axes (parallel to $\boldsymbol{e}_{\mathbf{3}}$ ) and their orientation is defined by a single angle $\beta^{(r)}$ (see figure $5)$. When such columnar polycrystals are loaded by an antiplane shear strain : 


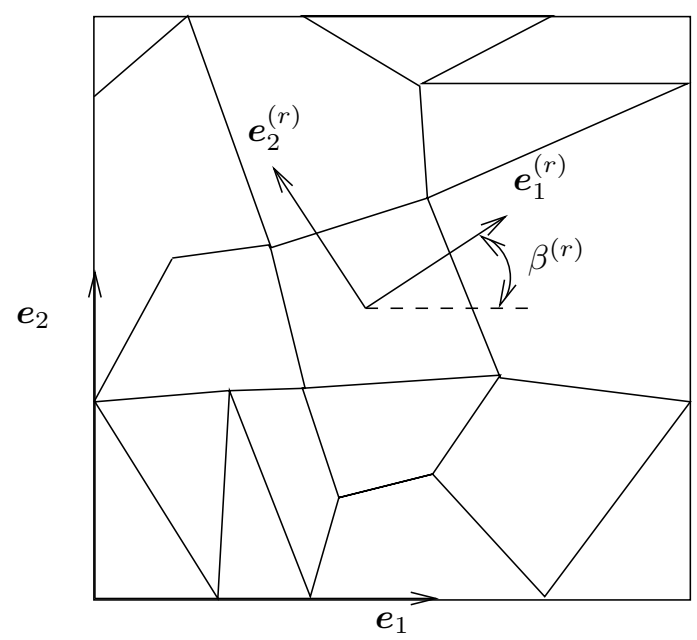

Figure 5: 2D polycrystals.

$$
\bar{\varepsilon}=\bar{\varepsilon}_{13}\left(\boldsymbol{e}_{\mathbf{1}} \otimes \boldsymbol{e}_{\mathbf{3}}+\boldsymbol{e}_{\mathbf{3}} \otimes \boldsymbol{e}_{\mathbf{1}}\right)+\bar{\varepsilon}_{23}\left(\boldsymbol{e}_{\mathbf{2}} \otimes \boldsymbol{e}_{\mathbf{3}}+\boldsymbol{e}_{\mathbf{3}} \otimes \boldsymbol{e}_{\mathbf{2}}\right),
$$

the only two slip systems that can be activated are defined by :

$$
\boldsymbol{\mu}_{(1)}=\frac{1}{2}\left(e_{1} \otimes e_{3}+e_{3} \otimes e_{1}\right) \quad \text { et } \quad \mu_{(2)}=\frac{1}{2}\left(e_{2} \otimes e_{3}+e_{3} \otimes e_{2}\right) .
$$

The boundary value problem (2) is two dimensional and the only non-zero components of the strain and stress tensors are $\varepsilon_{13}, \varepsilon_{23}, \sigma_{13}$ et and $\sigma_{23}$ which are functions of $x_{1}$ and $x_{2}$. Moreover if the crystal have orthotropic symmetry, the only components of the elastic stiffness moduli tensor which enter the boundary value problem are the shear moduli $L_{1313}$ and $L_{2323}$ denoted $g_{(1)}$ et and $g_{(2)}$ in the sequel. Then, $\boldsymbol{M}^{\mathrm{e}}$ is defined as :

$$
\boldsymbol{M}^{\mathrm{e}}=\left(2 g_{(1)}\right)^{-1} \boldsymbol{G}_{(1)}+\left(2 g_{(2)}\right)^{-1} \boldsymbol{G}_{(2)}, \quad \text { with } \quad \boldsymbol{G}_{(i)}=2\left(\boldsymbol{\mu}_{(i)} \otimes \boldsymbol{\mu}_{(i)}\right) .
$$

The tensors $\boldsymbol{G}_{(i)}$ have the following properties :

$$
\boldsymbol{G}_{(i)}: \boldsymbol{G}_{(j)}=\delta_{i j} \boldsymbol{G}_{(i)}, \boldsymbol{G}_{(i)}: \boldsymbol{\mu}_{(i)}=\boldsymbol{\mu}_{(i)} \text { and } \boldsymbol{\mu}_{(i)}: \boldsymbol{\mu}_{(i)}=\frac{1}{2} \text {. }
$$

Note that the constitutive behavior (26) belongs to the more general class of Maxwellian solids (1) with a viscous compliance tensor $\boldsymbol{M}^{\mathrm{v}(r)}$ defined by :

$$
\boldsymbol{M}^{\vee(r)}=\sum_{k=1}^{K}\left(2 g_{(k)}^{\vee}\right)^{-1} \boldsymbol{G}_{(k)}^{(r)}, \quad \text { with } \quad \boldsymbol{G}_{(i)}^{(r)}=2\left(\boldsymbol{\mu}_{(i)}^{(r)} \otimes \boldsymbol{\mu}_{(i)}^{(r)}\right) \quad \text { and } \quad g_{(k)}^{\vee}=\frac{\sigma_{0,(k)}}{\dot{\gamma}_{0,(k)}}
$$

\subsection{The RVP estimate applied to $2 D$ polycrystals}

Consider a 2D polycrystal made of $N$ grains characterized by the elastic and the viscous compliance moduli (29) and (30). As in section 3, the tensor $\boldsymbol{H}^{(r)}$ in the $r$-th grain is chosen proportional to the identity tensor :

$$
\boldsymbol{H}^{(r)}=h^{(r)} \boldsymbol{I},
$$

This choice leads to the following tensors for the local problem (6) :

$$
\left.\begin{array}{l}
\mathcal{L}_{0}^{(r)}=\sum_{k=1}^{2} 2 g_{\Delta,(k)}^{(r)} \boldsymbol{G}_{(k)}^{(r)}, \quad g_{\Delta,(k)}=\frac{g_{(k)} \Delta t g_{(k)}^{\vee}}{g_{(k)} \Delta t+g_{(k)}^{\vee}\left(1-h^{(r)}\right)} \\
\boldsymbol{\tau}_{0}^{(r)}=\sum_{k=1}^{2} \frac{g_{(k)}^{\vee}\left(1-h^{(r)}\right)}{g_{(k)} \Delta t+g_{(k)}^{\vee}\left(1-h^{(r)}\right)} \boldsymbol{G}_{(k)}^{(r)}: \boldsymbol{\sigma}_{n}^{(r)} .
\end{array}\right\}
$$


$\boldsymbol{\sigma}_{n}^{(r)}$ and $h^{(r)}$ are determined thanks to the specific form taken by the closure relations (10) and (17), given the choice (31). The relations (7) and (17) become respectively :

$$
\left.\begin{array}{c}
\left\langle\boldsymbol{\sigma}_{n}\right\rangle_{r}=h^{(r)}\langle\boldsymbol{\sigma}\rangle_{r}+\left(1-h^{(r)}\right) \boldsymbol{\sigma}_{n}^{(r)}, \\
\boldsymbol{C}^{(r)}\left(\boldsymbol{\sigma}_{n}\right)::\left(\sum_{k=1}^{2} \frac{1}{2 g_{(k)}} \boldsymbol{G}_{(k)}^{(r)}\right)=\left(h^{(r)}\right)^{2} \boldsymbol{C}^{(r)}(\boldsymbol{\sigma})::\left(\sum_{k=1}^{2} \frac{1}{2 g_{(k)}} \boldsymbol{G}_{(k)}^{(r)}\right) .
\end{array}\right\}
$$

The first and second moments of the field $\boldsymbol{\sigma}$ solution of the boundary value problem (5) are estimated by the self-consistent model. For the checkerboard polycrystal (see figure 6 ), this model gives the exact value of the effective elastic shear modulus $\left(\tilde{g}=\sqrt{g_{(1)} g_{(2)}}\right)$ and of the effective viscosity $\left(\tilde{g}^{\vee}=\sqrt{g_{(1)}^{\vee} g_{(2)}^{\vee}}\right)$ (see Lebensohn et al., 2005).

The resolution of the system of equations (33) is discussed in Appendix B. As for two-phase composites, the second equation in (33) may have several roots. But unlike in the case of twophase composites, no heuristic procedure for selecting the most physical root has been found by the authors when the number of grains (or equivalently of unknowns $h^{(r)}$ ) is large. This is why the results presented below for random polycrystals are restricted to monotonic loadings for which the system (33) has a single set of roots.

As in section 3, the simple choice (31) for $\boldsymbol{H}^{(r)}$ can be questioned. Other forms have been explored, in particular $\boldsymbol{H}^{(r)}=h_{1}^{(r)} \boldsymbol{G}_{(1)}+h_{2}^{(r)} \boldsymbol{G}_{(2)}$. No significant improvement was observed and the corresponding results are not shown here.

\subsection{Untextured random polycrystals}

The polycrystals considered in this section are composed of equiaxed, randomly oriented grains. The accuracy of the model is assessed by comparison with reference results obtained by full-field simulations performed with a computational method based on Fast Fourier Transforms, originally proposed by Moulinec and Suquet (1998) for composites and extended by Lebensohn (2001) to polycrystals. The reference results shown here were obtained using the freeware CRAFT (freely available at http://craft.lma.cnrs-mrs.fr). The microstructures are Voronoi tessellations in which the grains are randomly oriented (defined by a single angle $\beta$ between 0 and $\pi$ ). The macroscopic behavior of such polycrystals tends to be isotropic when the number of grains is increased. A typical microstructure is shown in figure 6 . All computations are performed on 8 different configurations containing 2000 grains each, discretized over a $4096 \times 4096$ Fourier grid. Ensemble averages of the results are taken by the following relation (as in Lebensohn et al., 2005) (with $N_{c}=8, N_{c}$ being the number of configurations) :

$$
\bar{f}=\frac{1}{N_{c}} \sum_{j=1}^{N_{c}}\langle f\rangle^{j}, \quad \text { with }\langle f\rangle^{j} \text { the average of } f \text { in the } j-\text { th configuration. }
$$

The first and second moments are defined for 10 different angular sectors $I$ (between 0 and $\pi$ ) as:

$$
\{f\}_{I}=\frac{1}{N_{c}} \sum_{j=1}^{N_{c}} \sum_{i} \frac{c^{(i)}}{c^{I}}\langle f\rangle_{i}^{j} \quad \text { and } \quad\left\{f^{2}\right\}_{I}=\frac{1}{N_{c}} \sum_{j=1}^{N_{c}} \sum_{i} \frac{c^{(i)}}{c^{I}}\left\langle f^{2}\right\rangle_{i}^{j} \quad \text { with } \quad c^{I}=\sum_{i} c^{(i)}
$$

where $\langle f\rangle_{i}^{j}$ is the average of $f$ over grain $i$ in the $j$-th configuration, and where the summation over $i$ extends over all grains $i$ whose orientation, defined by the angle $\beta^{(i)}$, is in the following angular sector :

$$
\frac{\pi(I-1)}{10} \leq \beta^{(i)}<\frac{\pi I}{10} \quad \text { and } \quad I=1, \ldots, 10 .
$$


The average of the stress fluctuations is defined as :

$$
\boldsymbol{C}^{I}(\boldsymbol{\sigma}):: \boldsymbol{K}=2\left\{\left(\sigma_{13}-\left\{\sigma_{13}\right\}_{I}\right)^{2}+\left(\sigma_{23}-\left\{\sigma_{23}\right\}_{I}\right)^{2}\right\}_{I}
$$

The predictions of the RVP estimate are obtained by computing the first and the second moment of the stress field (solution of the boundary value problem (6)) by the self-consistent method with 40 families $i$ of grains, with the same volume fraction $c^{(i)}=1 / 40$ and with orientation $\beta^{(i)}=\frac{(i-1) \pi}{40}$ (to ensure macroscopic isotropy of the polycrystal). The first moment and the fluctuations of the stress field given by the RVP estimate (using the self-consistent model) are compared with the ensemble averages of the full-field calculations according to the relations (35) and (36).
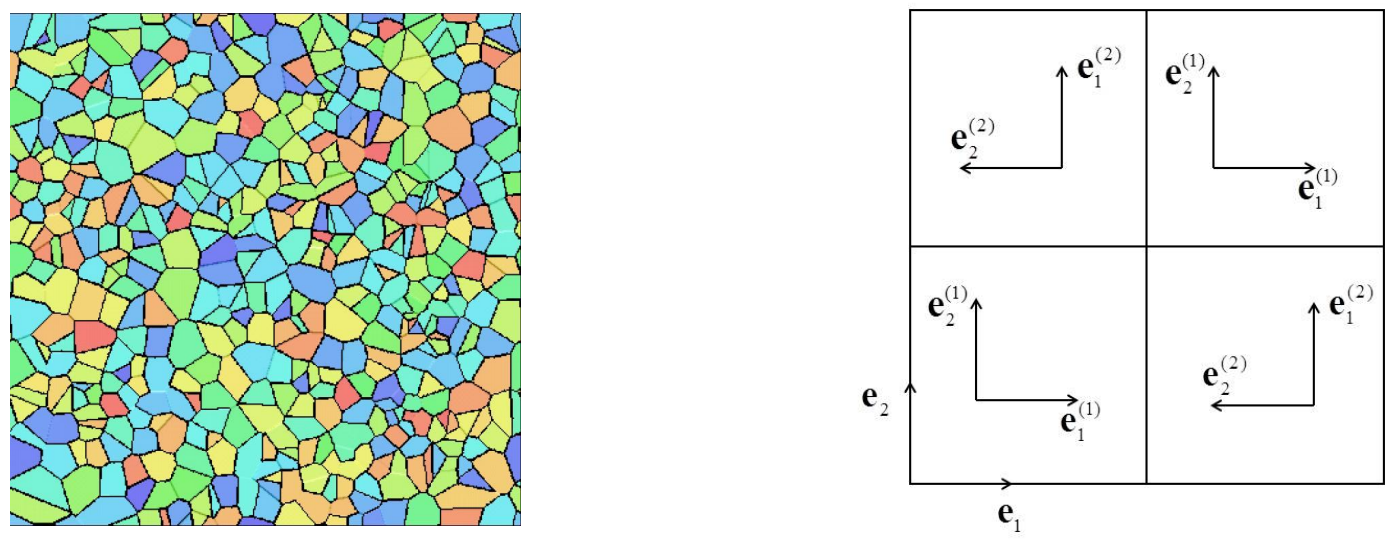

Figure 6: 2D polycrystals. Left: Voronoi tessellation. Right: checkerboard

The mechanical characteristics of the single crystal are :

$$
g_{(1)}=1 \mathrm{MPa}, \quad g_{(2)}=1 \mathrm{MPa}, \quad g_{(1)}^{\vee}=1 \mathrm{MPa} \cdot \mathrm{s}, \quad g_{(2)}^{\vee}=400 \mathrm{MPa} \cdot \mathrm{s},
$$

corresponding to relaxation times $\tau_{(1)}=1 \mathrm{~s}$ and $\tau_{(2)}=400 \mathrm{~s}$ for the different slip systems.

Two loadings are considered: a monotonic simple shear test (at constant strain-rate) and a creep test at two different levels of stress.

Monotonic simple shear test at constant strain-rate. The imposed overall strain is :

$$
\bar{\varepsilon}(t)=\dot{\bar{\varepsilon}}_{13} t\left(e_{1} \otimes e_{3}+e_{3} \otimes e_{1}\right) \quad \text { with } \quad \dot{\bar{\varepsilon}}_{13}=5.10^{-1} s^{-1}
$$

The macroscopic stress, the first moment and the fluctuations of the stress field estimated by the RVP model and obtained by the full-field calculations are compared in figure 7 . The statistics of the stress field (first moment and fluctuations) as a function of the grain orientation are shown at 3 specific times corresponding to $t_{1}=1 \mathrm{~s}, t_{2}=200 \mathrm{~s}$ et and $t_{3}=1200 \mathrm{~s}$ (these 3 specific times are shown by 3 circles in figure 7 (a)). At time $t_{1}$, the polycrystal behavior is purely elastic (instantaneous response), at time $t_{3}$, it is purely viscous (long time response). At time $t_{2}$ elasticity and viscosity are coupled (transient response).

Although the contrast between the relaxation times of the slip systems is large $\left(\tau_{(2)}=400 \tau_{(1)}\right)$, the macroscopic response is very well estimated by the RVP model (the maximum error is less than 
$2 \%$ ). At the 3 specific times, the RVP model gives a very accurate estimate of the first moment of the stress field as a function of the grain orientation. The RVP model predicts a constant stress fluctuation (no dependence on the grain orientation), which is consistent with the results of the FFT simulations (the small variations could be induced by the finite number of grains used in the computations).

The independence of the fluctuations on the orientation is exactly predicted by the selfconsistent scheme in the purely elastic or purely viscous limits. Indeed it is found the average and standard deviations of the resolved shear stresses in a $2 \mathrm{~d}$ polycrystal given in Lebensohn et al. $(2004)^{2}$ that with the self-consistent scheme :

$$
\sqrt{\frac{C^{I}(\boldsymbol{\sigma}):: \boldsymbol{K}}{\overline{\boldsymbol{\sigma}}: \overline{\boldsymbol{\sigma}}}}=\frac{|\sqrt{M}-1|}{\sqrt{M}+1} \sqrt{\frac{M+1}{2 \sqrt{M}}},
$$

where $M$ is the contrast of the moduli (elastic or viscous) in the two directions $M=\mu_{1} / \mu_{2}$. In the present example, the contrast in the purely viscous regime is $M=400$ and the ratio (37) is found equal to 2.86 which is consistent with the fluctuations observed in figure $7(\mathrm{c})$, both for the RVP model and the FFT simulations, when $t=t_{3}$.

The fluctuations of the stress field increase with time: at time $t_{1}$, there is no fluctuations since there is no contrast in the elastic moduli and at time $t_{3}$ these fluctuations are large because of the important contrast between the viscosities of the slip systems. This evolution of the fluctuations during the simulation is very accurately captured by the model with a maximum error of about $8 \%$.

Creep test with stress discontinuity. The macroscopic imposed stress is defined as:

$$
\overline{\boldsymbol{\sigma}}=\bar{\sigma}_{13}(t)\left(\boldsymbol{e}_{1} \otimes \boldsymbol{e}_{3}+\boldsymbol{e}_{3} \otimes \boldsymbol{e}_{1}\right)
$$

with

$$
\bar{\sigma}_{13}(t)=\left\{\begin{array}{lll}
1 & \text { when } t<2000 \mathrm{~s} \\
2 & \text { when } t>2000 \mathrm{~s}
\end{array}\right.
$$

The RVP estimates and the full-field computations are shown in figure 8. Again a good accuracy of the RVP model for the estimates of the macroscopic behavior and the statistics of the stress field (first moment and fluctuations for 3 time values) is observed.

\subsection{Checkerboard}

This section is devoted to the two-phase checkerboard (see Milton, 2002; Lebensohn et al., 2005). This 2D polycrystal (shown on figure 6), is composed of two phases (with the same volume fraction $c^{(1)}=c^{(2)}=0.5$ ), distributed in 4 grains repeated periodically. The orientation of phase (1) is $\beta^{(1)}=0$ and that of phase (2) is $\beta^{(2)}=\frac{\pi}{2}$. The reference results used to assess the accuracy of the RVP model are obtained with full-field simulations (FFT) on a $4096 \times 4096$ Fourier grid. The RVP estimates are obtained with the exact analytical result for the checkerboard. The polycrystal is subjected to the following macroscopic strain :

$$
\bar{\varepsilon}(0)=0, \dot{\bar{\varepsilon}}(t)= \pm \dot{\bar{\varepsilon}}_{0}\left(\boldsymbol{e}_{1} \otimes \boldsymbol{e}_{3}+\boldsymbol{e}_{3} \otimes \boldsymbol{e}_{1}\right), \dot{\bar{\varepsilon}}_{0}=5 \cdot 10^{-1} \mathrm{~s}^{-1} .
$$

Different set of elastic and viscous shear moduli are considered :

- $g_{(1)}=10 \mathrm{MPa}, g_{(2)}=10 \mathrm{MPa}, g_{(1)}^{\mathrm{v}}=0.2 \mathrm{MPa.s}$ and $g_{(2)}^{\mathrm{v}}=20 \mathrm{MPa} . \mathrm{s}$.

- $g_{(1)}=10 M P a, g_{(2)}=100 M P a, g_{(1)}^{\vee}=0.2 M P a . s$ and $g_{(2)}^{\vee}=20 M P a . s$.

\footnotetext{
${ }^{2}$ Note however that their expression for $\bar{\tau}_{2}^{(r)}$ should be multiplied by $\sqrt{M}$, but that does not affect the fluctuations
} 


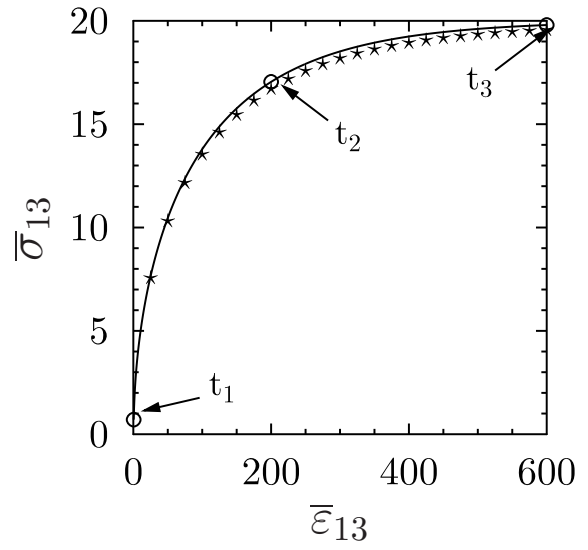

(a)

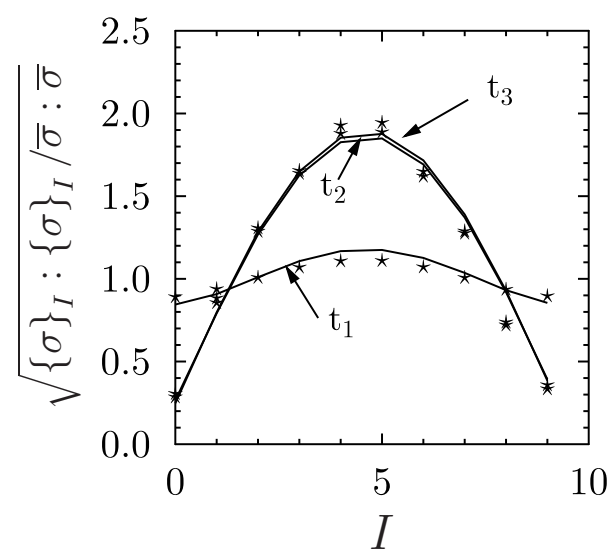

(b)

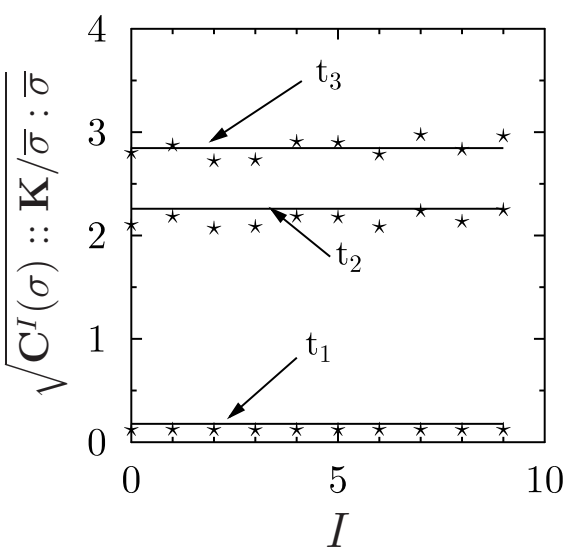

(c)

Figure 7: Random polycrystal under shear at constant strain-rate. Comparison between the RVP model (solid line) and the full-field (FFT) simulations (symbols). (a) Macroscopic stress $\bar{\sigma}_{13}$. The three time steps for which the statistics of the stress field are compared are shown by circles. (b) Norm of the first moment of the stress as a function of the grain orientation. (c) Stress fluctuations as a function of the grain orientation. 


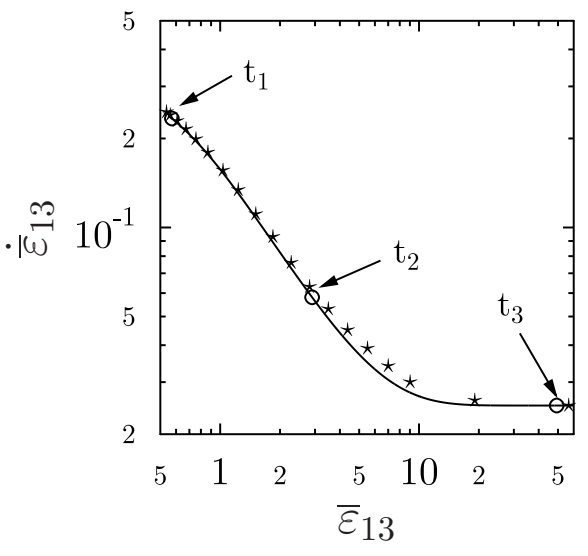

(a)

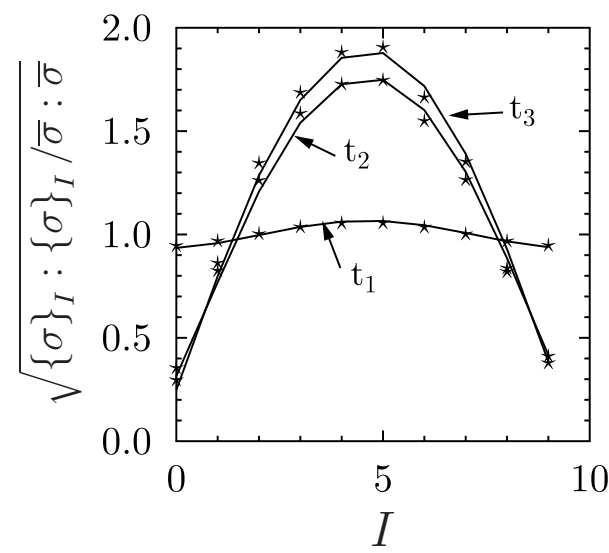

(c)

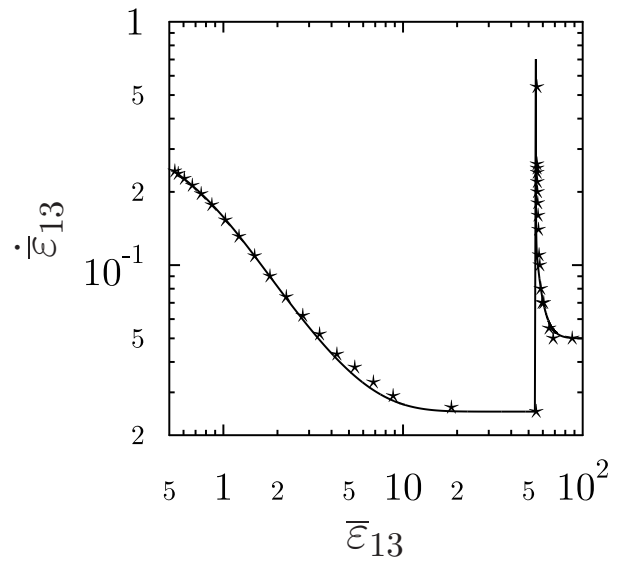

(b)

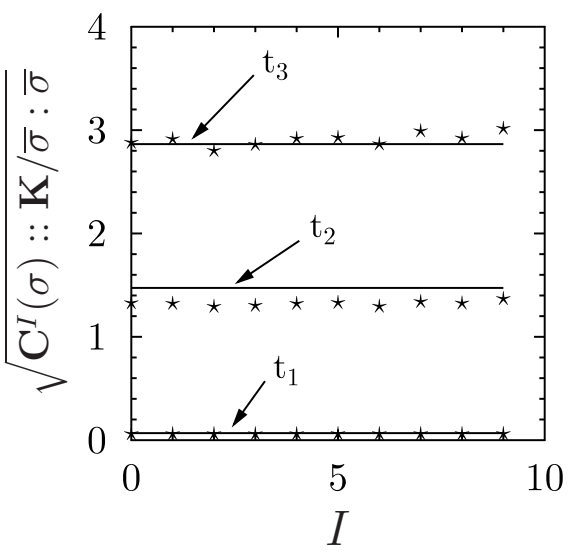

(d)

Figure 8: Random polycrystal subjected to a creep test. Comparison between the RVP model (solid line) and the full-field (FFT) simulations (symbols). (a) Effective response in the first loading stage $\left(\sigma_{13}=1 M P a\right)$ and the three time steps for which the statistics of the stress field are compared (circles).(b) Effective behavior in the first and the second loading stages $\left(\sigma_{13}=1 \mathrm{MPa}\right.$ and $\left.\sigma_{13}=2 \mathrm{MPa}\right)$. (c) Norm of the first moment of the stress as a function of the grain orientation. (d) Stress fluctuations as a function of the grain orientation. 
- $g_{(1)}=10 \mathrm{MPa}, g_{(2)}=100 \mathrm{MPa}, g_{(1)}^{\vee}=20 \mathrm{MPa} . \mathrm{s}$ and $g_{(2)}^{\vee}=20 \mathrm{MPa} . \mathrm{s}$.

The overall response predicted by by the RVP model is compared in figure 9 with the reference results (full-field FFT simulations). These plots call for the following comments.

- In the first part of the loading history $(t<2 s)$, the RVP estimate is very accurate for all material parameters, the maximum error is less than $1 \%$.

- The accuracy of the model is poorer in the transient zone when the elastic and the viscous effects are coupled (just after the inversion of the loading direction, for $t>2 s$ and $t>6 s$ ).

- The discrepancy between the macroscopic stress predicted by the proposed model and the reference results increases when the transient zone is extended and when the viscous contrast is large.

\section{Conclusion}

A simplified derivation of the variational estimate RVP of Lahellec and Suquet (2013) has been presented for linear viscoelastic composites. This estimate not only predicts the effective response of these composites, but also the first and second-order statistics of the local stress field in the different phases.

Using a step by step procedure is used (discretization of the time interval of study in different time step), the stress and strain fields at each time step are solution of a thermoelastic problem with a nonuniform eigenstrain. It is shown here that when this nonuniform eigenstrain is approximated by its average in each phase (first moment approximation, model RVP1st), the effective response of the composite is not accurately estimated.

In the proposed model (RVP), the nonuniform eigenstrain field is approximated by another field which has the two following merits :

(i) both fields have the same first and second moments (and not only the same first moment as in the model RVP1st),

(ii) the resulting problem is a classical thermoelastic problem (with a piecewise constant eigenstrain) and the statistics of the local fields can be estimated by classical homogenization schemes (Hashin-Shtrikman lower bound and self-consistent estimate for the applications considered in this paper).

The accuracy of the proposed method is assessed for two classes of composites (with Maxwellian phases):

(i) Two-phase particulate composites (with isotropic phases). The statistics of the local fields solutions of the boundary value problem (in the RVP model) are estimated by the HashinShtrikman lower bound and reference results (which are exact for such a microstructure) are obtained.

(ii) 2D columnar polycrystals under antiplane loading. The statistics of the local fields are estimated with the self-consistent estimate and the reference results are obtained by full field simulations (FFT).

These comparisons show that in two-phase particulate composites the RVP model gives a very good estimate of the effective behavior as well as of the first moments and the intraphase fluctuations of the local fields for both monotonic and cyclic loadings. By comparison, the RVP estimate is less accurate for polycrystals but is the only model available for predicting the higher-order statistics 


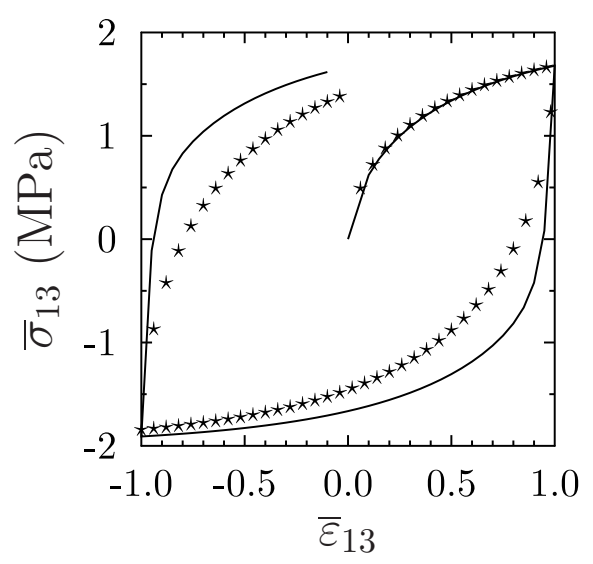

(a)

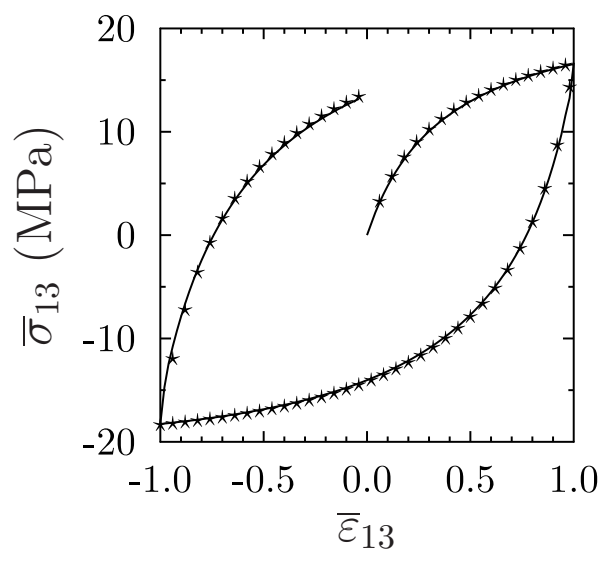

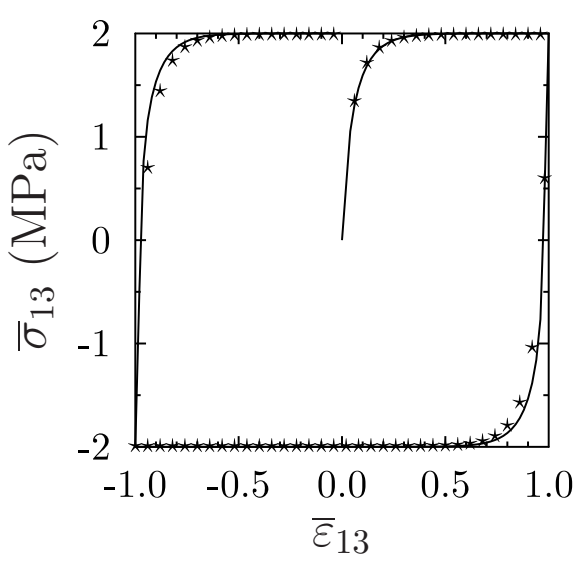

(b)

(c)

Figure 9: Checkerboard under cyclic loading. Comparison between the RVP model (solid line) and the full-field (FFT) simulations (symbols). (a) $g_{(1)}=10 \mathrm{MPa}, g_{(2)}=10 \mathrm{MPa}, g_{(1)}^{\vee}=0.2$ MPa.s and $g_{(2)}^{\mathrm{v}}=20$ MPa.s. (b) $g_{(1)}=10 \mathrm{MPa}, g_{(2)}=100 \mathrm{MPa}, g_{(1)}^{\mathrm{v}}=0.2$ MPa.s and $g_{(2)}^{\mathrm{v}}=20$ MPa.s. (c) $g_{(1)}=10 \mathrm{MPa}, g_{(2)}=100 \mathrm{MPa}, g_{(1)}^{\mathrm{v}}=20 \mathrm{MPa} . \mathrm{s}$ and $g_{(2)}^{\mathrm{v}}=20 \mathrm{MPa} . \mathrm{s}$. 
in viscoelastic media. For monotonic loadings (macroscopic strain or stress imposed) the effective behavior and the statistics of the local field are well predicted. For cyclic loadings, the possibility of mulitple roots is still an obstacle to a proper application of the method to polycrsyatls with a large number of different phases. For the checkerboard (a specific two-phase polycrystal, the RVP model can be applied but its predictions the effective response estimated by the RVP model can deviate from the reference results (depending on the material parameters) in the transient zone between elasticity and viscosity the purely elastic and the purely viscous regimes.

\section{Acknowledgments.}

This study was funded by the French "Agence Nationale de la Recherche" (project ELVIS, \#ANR-08-BLAN-0138). The authors also acknowledge the financial support of Labex MEC and of A*Midex through Grants ANR-11-LABX-0092 and ANR-11-IDEX-0001-02. 


\section{References:}

Diani, J., Gilormini, P., 2014. Using a pattern-based homogenization scheme for modeling the linear viscoelasticity of nano-reinforced polymers with an interphase. J. Mech. Phys. Solids 63, 51-61.

Francfort, G., Suquet, P., 1986. Homogenization and mechanical dissipation in thermoviscoelasticity. Arch. Rat. Mech. Anal. 96, 265-293.

Hashin, Z., 1970. Complex moduli of viscoelastic composites-I General theory and application to particulate composites. Int. J. Solids Structures 6, 539-552.

Idiart, M., Moulinec, H., Ponte Castañeda, P., Suquet, P., 2006. Macroscopic behavior and field fluctuations in viscoplastic composites: second-order estimates versus full-field simulations. J. Mech. Phys. Solids 54 , 1029-1063. Doi:10.1016/j.jmps.2005.11.004.

Idiart, M., Ponte Castañeda, P., 2007. Field statistics in nonlinear composites. I. Theory. Proc. R. Soc. London A 463, 183-202.

Lahellec, N., Suquet, P., 2007a. Effective behavior of linear vicoelastic composites: a timeintegration approach. Int. J. Sol. Struct. 44, 507-529. Doi:10.1016/j.ijsolstr.2006.04.038.

Lahellec, N., Suquet, P., 2007b. On the effective behavior of nonlinear inelastic composites: I. Incremental variational principles. J. Mech. Phys. Solids 55 , 1932-1963. Doi:10.1016/j/jmps.2007.02.003.

Lahellec, N., Suquet, P., 2007c. On the effective behavior of nonlinear inelastic composites: II. A second-order procedure. J. Mech. Phys. Solids 55, 1964-1992. Doi:10.1016/j/jmps.2007.02.004.

Laws, N., Mc Laughlin, R., 1978. Self-consistent estimates for the viscoelastic creep compliance of composite materials. Proc. R. Soc. London A 359, 251-273.

Lebensohn, R., 2001. N-site modelling of a 3D viscoplastic polycrystal using Fast Fourier Transforms. Acta Mater. 49, 2723-2737.

Lebensohn, R., Liu, Y., Ponte Castañeda, P., 2004. Macroscopic properties and field fluctuations in model power-law polycrystals: full-field solutions versus self-consistent estimates . Proc. R. Soc. London. A 460, 1381-1405.

Lebensohn, R., Castelnau, O., Brenner, R., Gilormini, P., 2005. Study of the antiplane deformation of linear 2-d polycrystals with different microstructures. Int. J. Sol. Struct. 42, 5441-5459. Doi:10.1016/j.ijsolstr.2006.04.038.

Liu, Y., Ponte Castañeda, P., 2004. Second-order theory for the effective behavior and field fluctuations in viscoplastic polycrystals. J. Mech. Phys. Solids 52 , 467-495.

Masson, R., Brenner, R., Castelnau, O., 2012. Incremental homogenization approach for ageing viscoelastic polycrystals. C.R. Mécanique 340, 378-386.

Mercier, S., Jacques, N., Molinari, A., 2005. Validation of an interaction law for the Eshelby inclusion problem in elasto-viscoplasticity. Int. J. Solids Struct. 42, 1923-1941.

Molinari, A., 2002. Averaging models for heterogeneous viscoplastic and elastic-viscoplastic materials. Journal of Engineering Materials and Technology 124, 62-70.

Ponte Castañeda, P., 1992. New variational principles in plasticity and their application to composite materials. J. Mech. Phys. Solids 40, 1757-1788. 
Ponte Castañeda, P., 2002a. Second-order homogenization estimates for nonlinear composites incorporating field fluctuations. I - Theory. J. Mech. Phys. Solids 50, 737-757.

Ponte Castañeda, P., 2002b. Second-order homogenization estimates for nonlinear composites incorporating field fluctuations. II - Applications. J. Mech. Phys. Solids 50, 759-782.

Ponte Castañeda, P., Suquet, P., 1998. Nonlinear composites, in: der Giessen, E.V., Wu, T. (Eds.), Advances in Applied Mechanics. Academic Press, New York. volume 34, pp. 171-302.

Rekik, A., Brenner, R., 2011. Optimization of the collocation inversion method for the linear viscoelastic homogenization. Mechanics Research Communications 38, 305-308.

Ricaud, J., Masson, R., 2009. Effective properties of linear viscoelastic heterogeneous media: Internal variables formulation and extension to ageing behaviours. Int. J. Solids Structures 46, $1599-1606$.

Rougier, Y., Stolz, C., Zaoui, A., 1993. Représentation spectrale en viscoélasticité linéaire des matériaux hétérogènes. C.R. Acad. Sc. Paris, II 316, 1517-1522.

Sanchez-Hubert, J., Sanchez-Palencia, E., 1978. Sur certains problèmes physiques d'homogénéisation donnant lieu à des phénomènes de relaxation. C.R. Acad. Sc. Paris Série A 286, 903-906.

Schapery, R. A., 1962. Approximate methods of transform inversion for viscoelastic stress analysis, in: Proc. U.S. Nat. Congr. Appl. Mech. ASME 4th. Vol. 2, 1075-1085.

Suquet, P., 1987. Elements of Homogenization for Inelastic Solid Mechanics, in: Sanchez-Palencia, E., Zaoui, A. (Eds.), Homogenization Techniques for Composite Media, Springer Verlag, New York. pp. 193-278.

Turner, P., Tomé, C., 1993. Self-consistent modeling of visco-elastic polycrystals: application to irradiation creep and growth. J. Mech. Phys. Solids 41, 1191-1211.

Vu, Q.H., Brenner, R., Castelnau, O., Moulinec, H., Suquet, P., 2012. A self-consistent estimate for linear viscoelastic polycrystals with internal variables inferred from the collocation method. Modelling and Simulation in Materials Science and Engineering 20, 024003. Doi:10.1088/09650393/20/2/024003.

Lahellec, N., Suquet, P., 2013. Effective response and field statistics in elasto-(viscoplastic) composites under radial and non-radial loadings. Int. J. Plasticity $42,1-30$.

Milton, G., 2002. The theory of composites. Cambridge University Press.

Moulinec, H., Suquet, P., 1998. A numerical method for computing the overall response of nonlinear composites with complex microstructure. Comp. Meth. Appl. Mech. Engng. 157, 69-94.

\section{Appendix A. First and second moments of the stress in particulate two-phase incom- pressible Maxwellian composites}

The aim of this appendix is to derive a set of differential equations satisfied by the first and the second moments of the stress field in a specific class of two-phase incompressible composites with overall isotropy made from incompressible, isotropic and Maxwellian phases. The microstructure of 
these composites are such that their linear properties are well described by one of Hashin-Shtrikman bounds. The constitutive relations of the phases read $\mathrm{as}^{3}$

$$
\dot{\varepsilon}=\frac{1}{2 \mu^{(r)}} \dot{s}+\frac{1}{2 \eta^{(r)}} \boldsymbol{s}=\frac{1}{2 \mu^{(r)}}\left(\dot{s}+\frac{1}{\tau^{(r)}} \boldsymbol{s}\right),
$$

where $\tau^{(r)}=\eta^{(r)} / \mu^{(r)}$ is the relaxation time of phase $r$. Upon application of the Laplace-Carson transform, these constitutive relations read in Laplace space (assuming that their initial state is stress-free and undeformed)

$$
\boldsymbol{s}^{*}(p)=2 \mu^{(r)^{*}}(p) \boldsymbol{\varepsilon}^{*}(p), \quad \mu^{(r)^{*}}(p)=\frac{\mu^{(r)}}{1+\frac{1}{\tau^{(r)} p}} .
$$

The microstructure of the composites is such that their effective linear properties (either purely elastic or purely viscous) are accurately described by the Hashin-Shtrikman estimate (phase 1 denotes the inclusions, phase 2 is the matrix):

$$
\tilde{\mu}\left(\mu^{(2)}, \mu^{(1)}\right)=\mu^{(2)}+c^{(1)} \frac{\mu^{(1)}-\mu^{(2)}}{1+c^{(2)} \beta \frac{\mu^{(1)}-\mu^{(2)}}{\mu^{(2)}}}, \quad \beta=\frac{2}{2+d},
$$

with a similar relation for $\tilde{\eta}$, and where $d=2$ or 3 is the dimension of space. As shown by Ricaud and Masson (2009) and Vu et al. (2012), the effective constitutive relations of these composites can be expressed with two internal variables only, as:

$$
\overline{\boldsymbol{s}}(t)=2 \bar{\mu}_{1}\left(\overline{\boldsymbol{\varepsilon}}(t)-\overline{\boldsymbol{\beta}}_{1}(t)\right)+2 \bar{\mu}_{2}\left(\overline{\boldsymbol{\varepsilon}}(t)-\overline{\boldsymbol{\beta}}_{2}(t)\right),
$$

where the evolution of the two tensorial internal variables $\overline{\boldsymbol{\beta}}_{1}$ and $\overline{\boldsymbol{\beta}}_{2}$ is governed by two differential equations

$$
\bar{\tau}_{i} \dot{\overline{\boldsymbol{\beta}}}_{i}(t)+\overline{\boldsymbol{\beta}}_{i}(t)=\overline{\boldsymbol{\varepsilon}}(t), \quad \overline{\boldsymbol{\beta}}_{i}(0)=0,
$$

involving the two relaxation times $\bar{\tau}_{2}$ and $\bar{\tau}_{1}$

$$
\left.\bar{\tau}_{1}=\tau^{(2)} \tau^{(1)} \frac{\mu^{(2)}\left(1-\beta c^{(2)}\right)+\mu^{(1)} \beta c^{(2)}}{\tau^{(2)} \mu^{(2)}\left(1-\beta c^{(2)}\right)+\tau^{(1)} \mu^{(1)} \beta c^{(2)}}, \quad \bar{\tau}_{2}=\tau^{(2)} .\right\}
$$

The corresponding weights $\bar{\mu}_{i}$ are given by:

$$
\left.\bar{\mu}_{1}=\mu^{(2)} \mu^{(1)} \frac{1-c^{(2)}}{\left(1-\beta c^{(2)}\right)} \frac{1}{\mu^{(2)}\left(1-\beta c^{(2)}\right)+\mu^{(1)} \beta c^{(2)}}, \quad \bar{\mu}_{2}=\mu^{(2)} \frac{c^{(2)}(1-\beta)}{1-\beta c^{(2)}} .\right\}
$$

\section{Appendix A.1. First moments}

The average strain in the inclusions in a linearly elastic composite is given by the HashinShtrikman estimate as:

$$
\overline{\boldsymbol{\varepsilon}}^{(1)}=a^{(1)} \overline{\boldsymbol{\varepsilon}}, \quad a^{(1)}\left(\mu^{(1)}, \mu^{(2)}\right)=\frac{1}{1+\beta c^{(2)}\left(\frac{\mu^{(1)}-\mu^{(2)}}{\mu^{(2)}}\right)} .
$$

\footnotetext{
${ }^{3}$ By incompressibility, the hydrostatic stress is left undetermined by the constitutive relations
} 
This relation can be applied with the moduli $\mu^{(r)^{*}}(p)$ in Laplace space to obtain the Laplace transform of the average strain and stress in the inclusions:

$$
\begin{aligned}
\overline{\boldsymbol{s}}^{(1)^{*}}(p) & =2 \mu^{(1)^{*}}(p) a^{(1)}\left(\mu^{(1)^{*}}(p), \mu^{(2)^{*}}(p)\right) \overline{\boldsymbol{\varepsilon}}^{*}(p) \\
& =\frac{1}{\frac{1}{\mu^{(1)} \tau^{(1)}}+\beta c^{(2)}\left(\frac{1}{\mu^{(2)} \tau^{(2)}}-\frac{1}{\mu^{(1)} \tau^{(1)}}\right)+p\left[\frac{1}{\mu^{(1)}}+\beta c^{(2)}\left(\frac{1}{\mu^{(2)}}-\frac{1}{\mu^{(1)}}\right)\right]} .
\end{aligned}
$$

Transforming back in real space it is found that the average stress in the inclusions is solution of the differential equation:

$$
\dot{\overline{\boldsymbol{s}}}^{(1)}(t)+\frac{1}{\bar{\tau}_{1}} \overline{\boldsymbol{s}}^{(1)}(t)=2 \bar{\mu}_{1} \frac{1-\beta c^{(2)}}{c^{(1)}} \dot{\overline{\boldsymbol{\varepsilon}}}(t) .
$$

This differential equation can be interpreted in two different, but complementary, ways. First, defining the purely elastic response of the inclusion to the macroscopic strain path $\bar{\varepsilon}(t)$ as:

$$
\overline{\boldsymbol{s}}_{e}^{(1)}(t)=2 \mu^{(1)} a^{(1)} \overline{\boldsymbol{\varepsilon}}(t), \quad a^{(1)}=a^{(1)}\left(\mu^{(1)}, \mu^{(2)}\right),
$$

(A.9) becomes, after some algebra,

$$
\dot{\overline{\boldsymbol{s}}}^{(1)}(t)+\frac{1}{\bar{\tau}_{1}} \overline{\boldsymbol{s}}^{(1)}(t)=\dot{\overline{\boldsymbol{s}}}_{e}^{(1)}(t),
$$

which confirms that for short times, the stress response is essentially elastic:

$$
t \simeq 0, \quad \overline{\boldsymbol{s}}^{(1)}(t) \simeq 0, \quad \dot{\boldsymbol{s}}^{(1)}(t) \simeq \dot{\overline{\boldsymbol{s}}}_{e}^{(1)}(t) .
$$

Second, defining the purely viscous response of the composite as

$$
\overline{\boldsymbol{s}}_{v}^{(1)}(t)=2 \mu_{v}^{(1)} a_{v}^{(1)} \dot{\bar{\varepsilon}}(t), \quad a_{v}^{(1)}=a^{(1)}\left(\eta^{(1)}, \eta^{(2)}\right),
$$

the differential equation (A.9) becomes, after some algebra,

$$
\bar{\tau}_{1} \dot{\overline{\boldsymbol{s}}}^{(1)}(t)+\overline{\boldsymbol{s}}^{(1)}(t)=\overline{\boldsymbol{s}}_{v}^{(1)}(t) .
$$

Therefore, for long times, if the overall strain-rate goes asymptotically to a limit $\dot{\bar{\varepsilon}}^{\infty}$, the stress response in the inclusions is essentially viscous and goes to a limit:

$$
t \rightarrow+\infty, \quad \dot{\boldsymbol{s}}^{(1)}(t) \rightarrow 0, \quad \overline{\boldsymbol{s}}^{(1)}(t) \rightarrow \overline{\boldsymbol{s}}_{v}^{(1)}(+\infty)=2 \mu_{v}^{(1)} a_{v}^{(1)} \dot{\overline{\boldsymbol{\varepsilon}}}^{\infty}
$$

The first moment of the stress in the matrix is simply obtained by the averaging rule:

$$
\overline{\boldsymbol{s}}^{(2)}(t)=\frac{1}{c^{(2)}}\left(\overline{\boldsymbol{s}}(t)-c^{(1)} \overline{\boldsymbol{s}}^{(1)}(t)\right) .
$$

Appendix A.2. Second moment of the stress

Define

$$
S^{(r)}(t)=\langle s(t): s(t)\rangle_{r} .
$$

In the inclusion phase, the stress delivered by the Hashin-Shtrikman estimate is uniform ${ }^{4}$

$$
S^{(1)}(t)=\overline{\boldsymbol{s}}^{(1)}(t): \overline{\boldsymbol{s}}^{(1)}(t),
$$

\footnotetext{
${ }^{4}$ This is a well-known result for elastic composites, which therefore also holds for viscoelastic composites after Laplace transform. The inverse Laplace transform does not affect the spatial homogeneity of the stress field in the inclusions.
} 
where $\overline{\boldsymbol{s}}^{(1)}(t)$ is known as solution of (A.9).

Then multiplying (A.1) by $\boldsymbol{\sigma}$ et integrating over the volume element yields, after proper use of Hill's relation:

$$
\overline{\boldsymbol{\sigma}}: \dot{\overline{\boldsymbol{\varepsilon}}}=\langle\boldsymbol{\sigma}: \dot{\boldsymbol{\varepsilon}}\rangle=\sum_{r=1}^{2} \frac{c^{(r)}}{2 \mu^{(r)}}\left(\langle\dot{\boldsymbol{s}}(t): \boldsymbol{s}(t)\rangle_{r}+\frac{1}{\tau^{(r)}}\langle\boldsymbol{s}(t): \boldsymbol{s}(t)\rangle_{r}\right)=\sum_{r=1}^{2} \frac{c^{(r)}}{4 \mu^{(r)}}\left(\dot{S}^{(r)}+\frac{2}{\tau^{(r)}} S^{(r)}\right),
$$

and after a few manipulations:

$$
\dot{S}^{(2)}+\frac{2}{\tau^{(2)}} S^{(2)}=\frac{4 \mu^{(2)}}{c^{(2)}} \overline{\boldsymbol{\sigma}}: \dot{\bar{\varepsilon}}-\frac{c^{(1)}}{c^{(2)}} \frac{\mu^{(2)}}{\mu^{(1)}}\left(\dot{S}^{(1)}+\frac{2}{\tau^{(1)}} S^{(1)}\right) .
$$

A differential equation for $S^{(2)}(t)$ is obtained. The right-hand-side, which is known, can be rearranged. Multiplying (A.9) by $\overline{\boldsymbol{s}}^{(1)}$ one gets

$$
\frac{1}{2} \dot{S}^{(1)}+\frac{1}{\bar{\tau}_{1}} S^{(1)}=2 \bar{\mu}_{1} \frac{1-\beta c^{(2)}}{c^{(1)}} \dot{\bar{\varepsilon}}: \overline{\boldsymbol{s}}^{(1)},
$$

and the final form of the differential equation for $S^{(2)}$ is:

$$
\dot{S}^{(2)}+\frac{2}{\tau^{(2)}} S^{(2)}=\frac{4 \mu^{(2)}}{c^{(2)}} \overline{\boldsymbol{\sigma}}: \dot{\bar{\varepsilon}}-\frac{4 \mu^{(2)}}{c^{(2)}} \frac{\bar{\mu}_{1}}{\mu^{(1)}}\left(1-\beta c^{(2)}\right) \overline{\boldsymbol{s}}^{(1)}: \dot{\bar{\varepsilon}}-2 \frac{c^{(1)}}{c^{(2)}} \frac{\mu^{(2)}}{\mu^{(1)}}\left(\frac{1}{\tau^{(1)}}-\frac{1}{\bar{\tau}_{1}}\right) S^{(1)} .
$$

In summary, the determination of $S^{(2)}(t)$ along a loading path requires first the integration of the differential equations (assuming that the initial state is stress-free and undeformed):

$$
\left.\begin{array}{rl}
\bar{\tau}_{1} \dot{\boldsymbol{\beta}}_{1}+\overline{\boldsymbol{\beta}}_{1} & =\overline{\boldsymbol{\varepsilon}}(t), \quad \overline{\boldsymbol{\beta}}_{1}(0)=0, \\
\bar{\tau}_{2} \dot{\boldsymbol{\beta}}_{2}+\overline{\boldsymbol{\beta}}_{2} & =\overline{\boldsymbol{\varepsilon}}(t), \quad \overline{\boldsymbol{\beta}}_{2}(0)=0, \\
\bar{\tau}_{1} \dot{\overline{\boldsymbol{s}}}^{(1)}+\overline{\boldsymbol{s}}^{(1)} & =\overline{\boldsymbol{s}}_{v}^{(1)}(t), \quad \overline{\boldsymbol{s}}^{(1)}(0)=0 .
\end{array}\right\}
$$

Then $\overline{\boldsymbol{s}}(t), \overline{\boldsymbol{s}}^{(1)}(t)$ and $S^{(1)}(t)$ are obtained from (A.4), (A.9) and (A.12). Finally $S^{(2)}(t)$ is obtained by integration of (A.14).

These integrations can be performed explicitly in simple situations. Consider for instance the case of macroscopic simple slip:

$$
\dot{\bar{\varepsilon}}(t)=\dot{\varepsilon}_{0}=\dot{\varepsilon}_{0} \boldsymbol{e}_{1} \otimes_{s} \boldsymbol{e}_{2}
$$

integration of (A.15) yields:

$$
\begin{aligned}
& \overline{\boldsymbol{s}}(t)=\overline{\boldsymbol{s}}_{v}\left(1-e^{-\frac{t}{\bar{\tau}_{1}}}\right)+\frac{2 \mu_{v}^{(2)} c^{(2)}(1-\beta)}{1-\beta c^{(2)}}\left(e^{-\frac{t}{\bar{\tau}_{1}}}-e^{-\frac{t}{\tau_{1}}}\right) \dot{\boldsymbol{\varepsilon}}_{0}, \\
& \overline{\boldsymbol{s}}^{(1)}(t)=\overline{\boldsymbol{s}}_{v}^{(1)}\left(1-e^{-\frac{t}{\bar{\tau}_{1}}}\right), \quad \overline{\boldsymbol{s}}_{v}=2 \tilde{\eta} \dot{\boldsymbol{\varepsilon}}_{0}, \quad \overline{\boldsymbol{s}}_{v}^{(1)}=2 \eta^{(1)} a_{v}^{(1)} \dot{\boldsymbol{\varepsilon}}_{0} .
\end{aligned}
$$

Then, integrating (A.14) leads to:

$$
\begin{aligned}
S^{(2)}(t) & =\mathcal{A}_{0} \frac{\tau^{(2)}}{2}\left(1-e^{-\frac{2 t}{\tau^{(2)}}}\right)+\mathcal{A}_{1} \tau^{(2)}\left(e^{-\frac{t}{\tau^{(2)}}}-e^{-\frac{2 t}{\tau^{(2)}}}\right) \\
& +\mathcal{A}_{2} \frac{\tau^{(2)} \bar{\tau}_{1}}{2 \bar{\tau}_{1}-\tau^{(2)}}\left(e^{-\frac{t}{\bar{\tau}_{1}}}-e^{-\frac{2 t}{\tau^{(2)}}}\right)+\mathcal{A}_{3} \frac{\tau^{(2)} \bar{\tau}_{1}}{2\left(\bar{\tau}_{1}-\tau^{(2)}\right)}\left(e^{-\frac{2 t}{\bar{\tau}_{1}}}-e^{-\frac{2 t}{\tau^{(2)}}}\right)
\end{aligned}
$$

where

$$
\begin{aligned}
& \mathcal{A}_{0}=\frac{4 \mu^{(2)}}{c^{(2)}}\left(\overline{\boldsymbol{s}}_{v}-c^{(1)} a_{v}^{(1)} \overline{\boldsymbol{s}}_{v}^{(1)}\right): \dot{\boldsymbol{\varepsilon}}_{0}, \quad \mathcal{A}_{1}=\frac{4 \mu^{(2)}}{c^{(2)}}\left(\overline{\boldsymbol{s}}_{v}+\frac{c^{(1)}}{1-\beta c^{(2)}} \overline{\boldsymbol{s}}_{v}^{(1)}\right): \dot{\boldsymbol{\varepsilon}}_{0}, \\
& \mathcal{A}_{2}=-\frac{4 \mu^{(2)}}{c^{(2)}} \frac{c^{(1)}}{1-\beta c^{(2)}} \overline{\boldsymbol{s}}_{v}^{(1)}: \dot{\boldsymbol{\varepsilon}}_{0}, \quad \mathcal{A}_{3}=-\frac{4 \mu^{(2)} c^{(1)}}{c^{(2)}} a_{v}^{(1)}\left(1-\frac{\tau^{(1)}}{\bar{\tau}_{1}}\right) \overline{\boldsymbol{s}}_{v}^{(1)}: \dot{\boldsymbol{\varepsilon}}_{0}
\end{aligned}
$$




\section{Appendix B. Determination of $\sigma_{n}^{(r)}$ and $h^{(r)}$}

$\boldsymbol{\sigma}_{n}^{(r)}$ and $h^{(r)}$ solve the set of equations :

$$
\left.\begin{array}{c}
\left\langle\boldsymbol{\sigma}_{n}\right\rangle_{r}=h^{(r)}\langle\boldsymbol{\sigma}\rangle_{r}+\left(1-h^{(r)}\right) \boldsymbol{\sigma}_{n}^{(r)}, \\
\boldsymbol{C}^{(r)}\left(\boldsymbol{\sigma}_{n}\right):: \boldsymbol{L}^{\mathrm{e}(r)^{-1}}=\left(h^{(r)}\right)^{2} \boldsymbol{C}^{(r)}(\boldsymbol{\sigma}):: \boldsymbol{L}^{\mathrm{e}(r)^{-1}} .
\end{array}\right\}
$$

These equations are the particular form of equations (10) and (11) when $\boldsymbol{H}^{(r)}$ is chosen proportional to the identity fourth-order tensor (ie $\left.\boldsymbol{H}^{(r)}=h^{(r)} \boldsymbol{I}\right)$. To avoid numerical difficulties, this set of equations is solved in two steps: first, $\boldsymbol{\sigma}_{n}^{(r)}$ is eliminated by the first equation of (B.1), and then, the second equation of (B.1) is solved numerically.

\section{Appendix B.1. Elimination of $\boldsymbol{\sigma}_{n}^{(r)}$}

Assuming the $h^{(r)}$ 's to be known for the time being, the first moment $\langle\boldsymbol{\sigma}\rangle_{r}$ of the stress in the individual phases of the LCC can be expressed as

$$
\langle\boldsymbol{\sigma}\rangle_{r}=\left(\mathcal{L}_{0}^{(r)}:\left(\boldsymbol{A}^{(r)}: \dot{\bar{\varepsilon}}+\boldsymbol{a}^{(r)}\right)+\boldsymbol{\tau}_{0}^{(r)}\right)
$$

where $\boldsymbol{A}^{(r)}$ is a $4^{\text {th }}$-order tensor depending on the microstructure, on $\mathcal{L}_{0}^{(r)}$ and the homogenization scheme used and $\boldsymbol{a}^{(r)}$ a $2^{\text {nd }}$-order tensor depending on $\left.\boldsymbol{A}^{(s)}\right|_{s=1, N}$ and $\left.\boldsymbol{\tau}_{0}^{(s)}\right|_{s=1, N}$ (It is important to note that this last dependency is linear). The first equation in (B.1) can be re-written as :

$$
\left\langle\boldsymbol{\sigma}_{n}\right\rangle_{r}=h^{(r)}\left(\mathcal{L}_{0}^{(r)}:\left(\boldsymbol{A}^{(r)}: \dot{\bar{\varepsilon}}+\boldsymbol{a}^{(r)}\right)+\boldsymbol{\tau}_{0}^{(r)}\right)+\left(1-h^{(r)}\right) \boldsymbol{\sigma}_{n}^{(r)}
$$

Assuming the $\left.h^{(s)}\right|_{s=1, N}$ to be known, (B.3) is a set of $N$ tensorial linear equations for $\left.\boldsymbol{\sigma}_{n}^{(r)}\right|_{r=1, N}$ which can be solved analytically ( $N$ is the number of phases).

Appendix B.2. Computation of $\left.h^{(s)}\right|_{s=1, N}$

The $\left.h^{(r)}\right|_{s=1, N}$ are determined by means of the second relation in (B.1). This relation makes use of the fluctuations of the stress field solution of the boundary value problem (6). These fluctuations are first related to the fluctuations of the strain-rate field with the elastic law (6) :

$$
\boldsymbol{C}^{(r)}(\boldsymbol{\sigma})=\mathcal{L}_{0}^{(r)}: \boldsymbol{C}^{(r)}(\dot{\varepsilon}): \mathcal{L}_{0}^{(r)} .
$$

To compute the fluctuations of the strain rate field, we introduce the following variational form of the boundary value problem (6) :

$$
\tilde{w}_{0}(\dot{\bar{\varepsilon}})=\operatorname{Inf}_{\langle\dot{\boldsymbol{\varepsilon}}\rangle=\dot{\bar{\varepsilon}}}\left\langle w_{0}(\dot{\boldsymbol{\varepsilon}})\right\rangle \quad \text { with } \quad w_{0}(\dot{\boldsymbol{\varepsilon}})=\frac{1}{2} \dot{\boldsymbol{\varepsilon}}: \mathcal{L}_{0}^{(r)}: \dot{\boldsymbol{\varepsilon}}+\boldsymbol{\tau}_{0}^{(r)}: \dot{\boldsymbol{\varepsilon}} .
$$

Following Idiart and Ponte Castañeda (2007), the fluctuations of the strain-rate field are then given by :

$$
\boldsymbol{C}^{(r)}(\dot{\boldsymbol{\varepsilon}})=\langle\dot{\boldsymbol{\varepsilon}} \otimes \dot{\boldsymbol{\varepsilon}}\rangle_{r}-\langle\dot{\boldsymbol{\varepsilon}}\rangle_{r} \otimes\langle\dot{\boldsymbol{\varepsilon}}\rangle_{r}=\frac{2}{c^{(r)}} \frac{\partial \tilde{w}_{0}}{\partial \mathcal{L}_{0}^{(r)}}-\langle\dot{\boldsymbol{\varepsilon}}\rangle_{r} \otimes\langle\dot{\boldsymbol{\varepsilon}}\rangle_{r} .
$$

At this stage of the computation of the $\left.h^{s)}\right|_{s=1, N}$, the particular form (class of symmetry) of the tensor $\mathcal{L}^{(r)}$ is accounted for in the second equation of (B.1). Two cases are considered here: isotropic and orthotropic (with $2 \mathrm{D}$ antiplane problem) tensors. 
Isotropic case: In the isotropic case, the second equation of (B.1) takes the simplified form of the second equation of $(21)$ which is rewritten as :

$$
\boldsymbol{C}^{(r)}\left(\boldsymbol{\sigma}_{n}\right)::\left(\frac{\boldsymbol{K}}{2 \mu^{(r)}}+\frac{\boldsymbol{J}}{3 k^{(r)}}\right)=\left(h^{(r)}\right)^{2} \boldsymbol{C}^{(r)}(\boldsymbol{\sigma})::\left(\frac{\boldsymbol{K}}{2 \mu^{(r)}}+\frac{\boldsymbol{J}}{3 k^{(r)}}\right) .
$$

Applying (B.4), this relation becomes :

$$
\boldsymbol{C}^{(r)}\left(\boldsymbol{\sigma}_{n}\right)::\left(\frac{\boldsymbol{K}}{2 \mu^{(r)}}+\frac{\boldsymbol{J}}{3 k^{(r)}}\right)=\left(h^{(r)}\right)^{2}\left(\langle\dot{\boldsymbol{\varepsilon}} \otimes \dot{\boldsymbol{\varepsilon}}\rangle_{r}-\langle\dot{\boldsymbol{\varepsilon}}\rangle_{r} \otimes\langle\dot{\boldsymbol{\varepsilon}}\rangle_{r}\right)::\left(\frac{2 \mu_{\Delta, 0}^{(r)}{ }^{2}}{\mu^{(r)}} \boldsymbol{K}+\frac{3 k_{\Delta, 0}^{(r)^{2}}}{k^{(r)}} \boldsymbol{J}\right)
$$

The projections of the second moment of the strain rate field on the tensors $\boldsymbol{K}$ and $\boldsymbol{J}$ are obtained by derivation of $\tilde{w}_{0}$ :

$$
\langle\dot{\boldsymbol{\varepsilon}} \otimes \dot{\boldsymbol{\varepsilon}}\rangle_{r}:: \boldsymbol{K}=\frac{1}{c_{r}} \frac{\partial \tilde{w}_{0}}{\partial \mu_{\Delta, 0}^{(r)}} \quad \text { and } \quad\langle\dot{\boldsymbol{\varepsilon}} \otimes \dot{\boldsymbol{\varepsilon}}\rangle_{r}:: \boldsymbol{J}=\frac{2}{3 c_{r}} \frac{\partial \tilde{w}_{0}}{\partial k_{\Delta, 0}^{(r)}} .
$$

2D antiplane polycrystals : In that case, the second equation of (B.1) takes the simplified form of the second equation of (33) which is rewritten as :

$$
\boldsymbol{C}^{(r)}\left(\boldsymbol{\sigma}_{n}\right)::\left(\sum_{k=1}^{2} \frac{1}{2 g_{(k)}} \boldsymbol{G}_{(k)}^{(r)}\right)=\left(h^{(r)}\right)^{2} \boldsymbol{C}^{(r)}(\boldsymbol{\sigma})::\left(\sum_{k=1}^{2} \frac{1}{2 g_{(k)}} \boldsymbol{G}_{(k)}^{(r)}\right) .
$$

Using (B.4), this relation becomes :

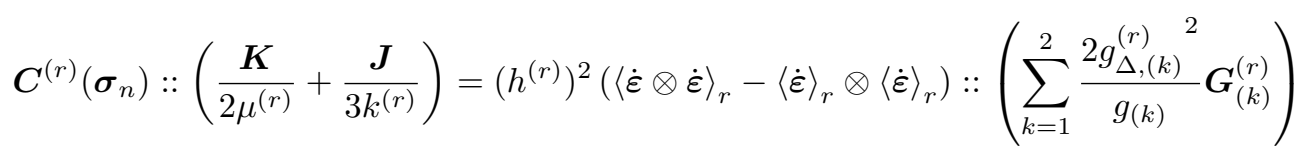

The projections of the second moment of the strain-rate field on the tensors $\boldsymbol{G}_{(k)}^{(r)}$ are obtained by derivation of $\tilde{w}_{0}$ :

$$
\langle\dot{\varepsilon} \otimes \dot{\varepsilon}\rangle_{r}:: \boldsymbol{G}_{(k)}^{(r)}=\frac{1}{c_{r}} \frac{\partial \tilde{w}_{0}}{\partial g_{\Delta,(k)}^{(r)}} .
$$

Appendix B.3. Two-phase particulate composites

For two-phase particulate composite with inclusions stiffer than the matrix the Hashin-Shtrikman lower bound is used to estimate the statistics of the strain rate field solution of the boundary value problem (6). This bound is also used to derive the reference results to avoid any possible error due to the homogenization scheme. This estimate predicts no fluctuation of the strain and stress fields in the inclusions. Therefore

$$
h^{(1)}=0 \quad \text { and } \quad \boldsymbol{\sigma}_{n}^{(1)}=\left\langle\boldsymbol{\sigma}_{n}\right\rangle_{1} .
$$

$h^{(2)}$ is solution of equation (B.8) which has to be solved numerically in the general case (closedform solutions can be found in very particular situations such as incompressible materials studied in section 3.2). Numerical difficulties are expected in the resolution of equation (B.8), which can be put in the form $f\left(h^{(2)}\right)=0$, where $f$ is a rational fraction with several poles. These poles come from the expression of $\boldsymbol{\sigma}_{n}^{(2)}$ resulting from (B.3) and from the expression of $\langle\boldsymbol{\sigma}\rangle_{2} . \boldsymbol{\sigma}_{n}^{(2)}$ is, again, a rational fraction with denominator $D E N(h)$.

In order to avoid the singularities due to the zeros of $D E N$ in the resolution of (B.8), this equation can be regularized by multiplying both sides by $D E N(h)$. The improvement is illustrated in figure B.10 where the variations with $h$ of $f$ before regularization (solid line) and after 
regularization (dashed line) are shown. These variations are shown for two different time steps, the first corresponding to $h<1$, i.e.

$$
\boldsymbol{C}^{(2)}\left(\boldsymbol{\sigma}_{n}\right)::\left(\frac{\boldsymbol{K}}{2 \mu^{(2)}}+\frac{\boldsymbol{J}}{3 k^{(2)}}\right)<\boldsymbol{C}^{(2)}(\boldsymbol{\sigma})::\left(\frac{\boldsymbol{K}}{2 \mu^{(2)}}+\frac{\boldsymbol{J}}{3 k^{(2)}}\right),
$$

and the other one corresponding to $h>1$, i.e.

$$
\boldsymbol{C}^{(2)}\left(\boldsymbol{\sigma}_{n}\right)::\left(\frac{\boldsymbol{K}}{2 \mu^{(2)}}+\frac{\boldsymbol{J}}{3 k^{(2)}}\right)>\boldsymbol{C}^{(2)}(\boldsymbol{\sigma})::\left(\frac{\boldsymbol{K}}{2 \mu^{(2)}}+\frac{\boldsymbol{J}}{3 k^{(2)}}\right) .
$$

Figure B.10 also shows that equation (B.8) can have several roots. (see figure B.10(b) for $h>1$ ). The two plots in this figure correspond to two different times along the loading and to two different situations for the nonlinear equation (B.8). In figure B.10(a), the equation has a single solution $h<1$ found as the intersection between the dotted line and the horizontal axis. By contrast, figure B.10(b) corresponding to a different time along the loading, two solutions are found. Unfortunately, there is no theoretical justification argument to discriminate the different solutions and a more pragmatic approach is used. In most cases (all the cases of section 3.3) the smallest positive value of $h^{(2)}$ is the best choice. In a few other cases (see section 3.2 for an example), when $\boldsymbol{C}^{(2)}(\boldsymbol{\sigma}):: \boldsymbol{L}^{\mathrm{e}^{(r)^{-1}}}$ (the left part of equation 17) decreases to very small values, the other solution has to be used to obtain the best estimate. Let $h_{0}=0, h_{1}$ be the smallest root of (B.8), $h_{2}$ be the largest root, $\overline{\boldsymbol{s}}\left(h_{i}\right)$ and $\boldsymbol{C}^{(2)}\left(\boldsymbol{\sigma}\left(h_{i}\right)\right)$ be respectively the average and the fluctuations of the stress field associated with the choice $h^{(2)}=h_{i}$. Then, $h^{(2)}$ is systematically taken equal to $h_{1}$, except when one of the two conditions (B.14) or (B.15) is met:

$$
\left.\begin{array}{l}
\boldsymbol{C}^{(2)}\left(\boldsymbol{\sigma}\left(h_{1}\right)\right)::\left(\frac{\boldsymbol{K}}{2 \mu^{(2)}}+\frac{\boldsymbol{J}}{3 k^{(2)}}\right)<\boldsymbol{C}^{(2)}\left(\boldsymbol{\sigma}_{n}\right)::\left(\frac{\boldsymbol{K}}{2 \mu^{(2)}}+\frac{\boldsymbol{J}}{3 k^{(2)}}\right) \\
\text { and } \overline{\boldsymbol{s}}^{(2)}\left(h_{1}\right)>\overline{\boldsymbol{s}}^{(2)}, \text { and } \overline{\boldsymbol{s}}\left(h_{1}\right): \overline{\boldsymbol{s}}\left(h_{1}\right)<\overline{\boldsymbol{s}}\left(h_{0}\right): \overline{\boldsymbol{s}}\left(h_{0}\right) . \\
\boldsymbol{C}^{(2)}\left(\boldsymbol{\sigma}\left(h_{1}\right)\right)::\left(\frac{\boldsymbol{K}}{2 \mu^{(2)}}+\frac{\boldsymbol{J}}{3 k^{(2)}}\right)<\boldsymbol{C}^{(2)}\left(\boldsymbol{\sigma}_{n}\right)::\left(\frac{\boldsymbol{K}}{2 \mu^{(2)}}+\frac{\boldsymbol{J}}{3 k^{(2)}}\right) \\
\text { and } \overline{\boldsymbol{s}}^{(2)}\left(h_{1}\right)<{\overline{\boldsymbol{s}_{n}}}^{(2)}, \text { and } \overline{\boldsymbol{s}}\left(h_{1}\right): \overline{\boldsymbol{s}}\left(h_{1}\right)>\overline{\boldsymbol{s}}\left(h_{0}\right): \overline{\boldsymbol{s}}\left(h_{0}\right) .
\end{array}\right\}
$$

\section{Appendix B.4. 2D polycrystals}

In untextured polycrystals, the statistics of the strain-rate field (and therefore the stress field) are estimated by the self-consistent method. For polycrystals with $N$ grains the $\left.h^{(s)}\right|_{s=1, N}$ are found by solving the set of $N$ nonlinear equations (B.11). Two classes of polycrystals are studied in section 4 :

i) Polycrystals with a large number of grains. The RVFP estimate can be implemented under monotonic load (constant rate of macroscopic stress or strain), there is only one set of $\left.h^{(s)}\right|_{s=1, N}$ verifying the conditions on the first and second moment of the stress. However, for cyclic loadings where there might be several solutions in each grain, we do not have a criterion for selecting the roots.

ii) Checkerboard. A checkerbopard is a specific two-phase composite. Therefore the RVP estimted can be implemented both for monotonic and cyclic loadings. Under cyclic loading it is observed that $\boldsymbol{C}^{(r)}(\boldsymbol{\sigma})::\left(\sum_{k=1}^{2} \frac{1}{2 g_{(k)}} \boldsymbol{G}_{(k)}^{(r)}\right)$ may decrease to very small values which makes equation (B.10) ill-conditioned. In this case, as for two-phase particulate composites, more than one set of $\left.h^{(s)}\right|_{s=1, N}$ may satisfy the equations and the choice between the different solutions is made by using the prescription of Appendix B.3. 


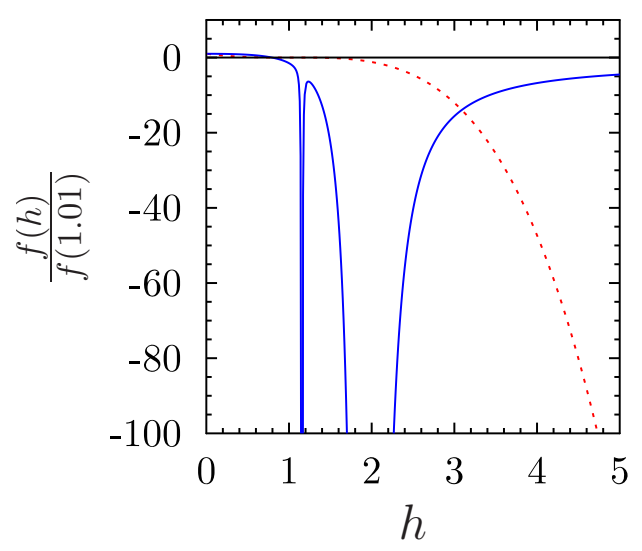

(a)

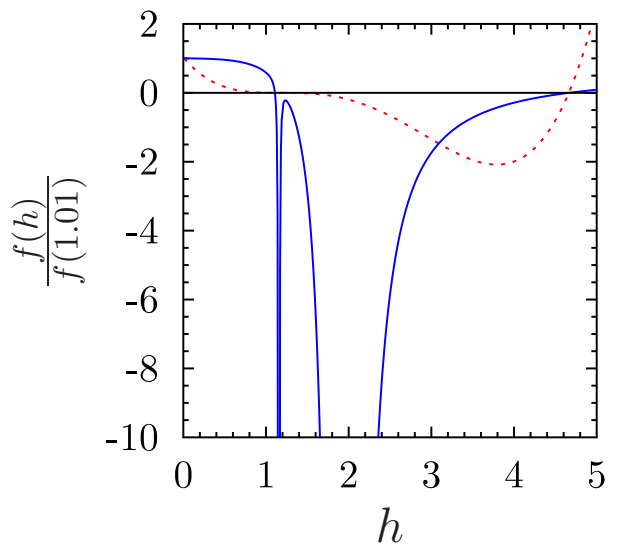

(c)

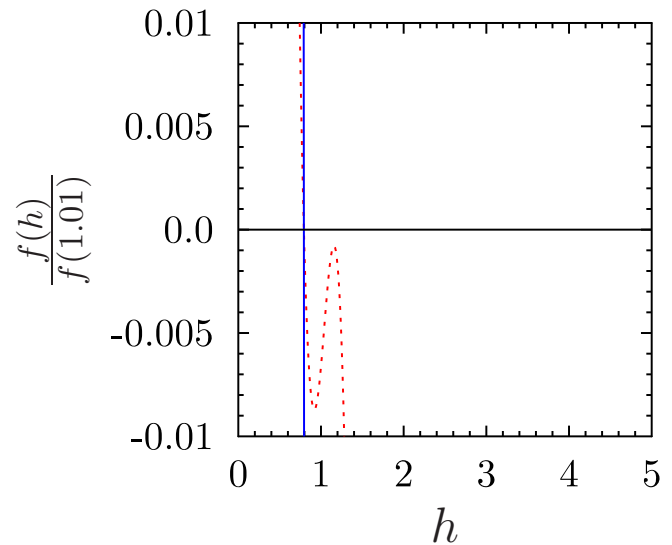

(b)

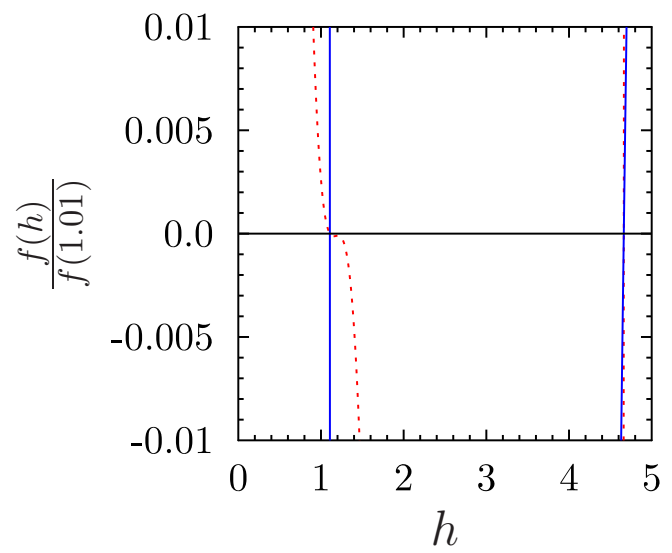

(d)

Figure B.10: Regularization of equation (B.8). Data as in figure 3 (c). Original function $f$ (solid line) compared with the regularized function (dashed line) as a function of $h$. (a) and (b) correspond to a situation where there is only one root $h<1$ to the equation $f=0$ (for $t=1 \mathrm{~s}$ ). (b) is a close-up of (a). (c) and (d) correspond to a situation where the equation $f=0$ has two roots $h>1$ (for $t=11 s$ ). (d) is a close-up of (c). 